\title{
Nutrigenetics and Dyslipidemia
}

\author{
Maryam Shalileh \\ Islamic Azad University of Hamedan Branch
}

Iran

\section{Introduction}

Dyslipidemia is an abnormal amount of lipids (abnormality in any of the lipoprotein fractions), especially elevated Low Density Lipoprotein (LDLs) and decreased High Density Lipoprotein (HDLs) in the blood (Shalileh et al., 2009). In developed countries, most dyslipidemias are hyperlipidemias; that is, an elevation of lipids in the blood, are often due to diet and lifestyle (Wikipedia., 2011; Shalileh et al., 2009). According to the Katharina studie's, elevated cholesterol levels and dyslipoproteinemia are metabolic abnormalities that are becoming increasingly significant in industrialized countries, but also worldwide (Shalileh et al., 2009).

There is a proportional increase in the risk of Coronary Heart Disease (CHD) with rising serum cholesterol levels (Shalileh et al., 2009). Dyslipidaemia is an important risk factor for Cardiovascular Disease (CVD) (Masson \& McNeili., 2005).

CVD is a common killer in both the Western and the developing world and is the leading cause of death globally (Lovegrove \& Gitau., 2008).

More people die annually from CVDs than from any other cause. An estimated 17. 1 million people died from CVDs in 2004, representing 29\% of all global deaths. Of these deaths, an estimated 7. 2 million were due to CHD and 5.7 million were due to stroke. By 2030, almost 23. 6 million people will die from CVDs, mainly from heart disease and stroke (WHO. 2011).

Atherosclerosis is the most common cause of CHD and related mortality (Debra., 2008; Shalileh et al., 2009) .Endothelial dysfunction initiates atherosclerosis (Shalileh et al., 2009) . The first observable event in the process of atherosclerosis is the accumulation of plaque (cholesterol from low-density lipoproteins, calcium, and fibrin) in the endothelium in large and medium arteries (Debra., 2008).

One of the factors that causes endothelial dysfunction is dyslipidemia (Shalileh et al., 2009).

\section{Treatment}

CVD represents the paradigm of multi factorial disorders encompassing multiple genetic and non modifiable risk factors, for example older age and modifiable risk factors such as elevated total and LDL-cholesteroland and triglycerides concentrations, reduced HDLcholesterol concentrations (Ordovas \& Corella., 2004; Ordovas., 2009; Perez-Martinez et al, 2011; Lovegrove \& Gitau, 2008). The interactions of those modulate plasma lipid concentrations and potentially CVD risk (Ordovas., 2009; Lovegrove \& Gitau., 2008). 
The link between serum cholesterol and the development of atherosclerosis was established a few decades ago and is now widely accepted. The National Cholesterol Education Program (NCEP) Adult Treatment Panel (ATP) publishes updated guidelines for treating lipid disorders. The latest version is the ATP III (Ordovas \& Corella., 2004; Debra., 2008).

The current recommendations aim to reduce the classical modifiable risk factors, and much emphasis has been placed on controlling high-plasma cholesterol levels (Ordovas \& Corella., 004).

Physicians are encouraged to refer patients to Registered Dietitians (RDs) to help patients meet goals for therapy (NCEP) based on LDL cholesterol levels. So the ATP III recommended the therapeutic lifestyle change (TLC) dietary pattern as the cornerstone for primary and secondary prevention of CHD. These guidelines consider dietary modification of treatment with emphasis on reducing the high saturated fat atherogenic diet and increased content of polyunsaturated fatty acid (PUFA) as well as controlling other behavioral factors. These therapies are used primarily to lower elevated blood levels of LDLC, raise HDL -C and lower triglycerides (TGs) (Rubin \& Berglund., 2002; Debra., 2008; Ordovas \& Orella., 2004).

\section{Response to the diet therapy}

Although dietary recommendations have been implemented to improve health and diminish the risk of CVD, type 2 diabetes and obesity, these recommendations have been established based on populations and not the individual. On the other hand the approach has been surprisingly unsuccessful in reducing CVD risk and the drastically different inter individual responses to a diet (Ordovas \& Corella., 2004).

So this clearly highlights the limitations of population-based nutritional recommendations and suggests that our understanding of the mechanisms responsible for inter-individual differences are far from being understood (Much et al., 2005).

Recent clinical evidence suggests dramatic inter-individual differences and existence of consistent hypo- and hyperresponders in response of plasma lipids to dietary manipulations, ranging from reduced LDL-C levels and TGs in some, to decreased HDL-C levels to elevated TGs. So in some, a low-fat diet has caused a shift to a lipid pattern that is more atherogenic than the original one. This supports the hypothesis that responsiveness is related to genetic variation and existence of nutrient-gene interactions or persons genotype (defined by the term 'nutrigenetics') (DeBusk, 2008; Perez-Martinez et al., 2011; Masson et al., 2003; Rideout., 2011; Ordovas et al., 2007).

A classic example of this is the large variation in the concentration of serum low-density lipoprotein-cholesterol (LDL-C) in response to fish oil supplementation. The cardio protective effects of the fatty acids in fish oil, Eicosapentaenoic Acid (EPA) and Docosahexaenoic Acid (DHA) are well recognized. However, a potentially deleterious increase in LDL-C (5-10\%) has been consistently reported after moderate to high doses of fish oil (>2 g day-1 EPA + DHA) (Lovegrove \& Gitau., 2008).

Despite this small, but significant increase in LDL-C, closer examination of the responses revealed a noticeable inter-individual variation. There was a mean increase in LDL-C of 4 . $1 \%$, yet the spread of individual responses was substantial, with 33 of the 74 subjects demonstrating a lower serum LDL-C and the remaining 41 demonstrating a higher LDL-C (range -40 to $+113 \%$ ) following fish oil intervention. This heterogeneous response to a change in dietary fat, may be attributed to a number of factors; including age, gender, 
baseline LDL-C levels, disease status and drug use. However, recent evidence strongly suggests that variations in a number of key genes may also be important (Lovegrove \& Gitau., 2008). For example, individuals with specific of genetic variants in a gene may experience different type of lipoprotein changes when placed on a particular diet, whereas individuals with other variants in the gene may be resistant to the effects of the same diet. Although data is sparse in regard to whether such interactions exist, some limited work suggests that interactions may play an important role in determining lipoprotein profiles and may thus be informative for CVD risk prediction. For example, knowledge of a patient's genetic information may allow medical providers and nutritional counselors to predict what lipoprotein changes are likely to occur if the patient starts a particular dietary intervention and, thus, better advise the patient regarding lifestyle changes (Musunuru., 2010; Ordovas \& Corella., 2004).

Lipoproteins are macromolecular complexes of lipids and proteins that originate mainly in the liver and intestine and are involved in transporting and redistributing lipids in the body. Lipid homeostasis is achieved by the coordinated action of numerous nuclear factors, enzymes, apolipoproteins, binding proteins, and receptors. Lipid metabolism is also linked with energy metabolism and is subject to many hormonal controls essential for adjusting to environmental and internal conditions. Genetic variability exists in humans for most of these components, and some of these mutations result in abnormal lipid metabolism and plasma lipoprotein profiles that may contribute to the pathogenesis of atherosclerosis. Many of these genes have been explored in terms of gene-diet interactions (Ordovas \& Corella., 2004).

So the shift towards personalized nutritional advice is a very attractive proposition, where, in principle, an individual can be given dietary advice specifically tailored to their genotype. However, the evidence-base for the impact of interactions between nutrients and fixed genetic variants on biomarkers of CVD risk is still very limited (Lovegrove \& Gitau., 2008; Ordovas., 2006; Masson et al., 2003; Masson \& Mc Neil., 2005; Fisler \& Warden., 2005).

With the advent of nutritional genomics, it's becoming clear that an individual's genetic makeup (genotype) is an important factor in this response and that dietary interventions must be matched to genotypes to effect the intended lipid-lowering responses (DeBusk., 2008).

A number of such genes have already been identified and include those involved with postprandial lipoprotein and triglyceride responses, homocysteine metabolism, hypertension, blood-clotting, and inflammation (Ordovas \& Corella., 2004; DeBusk., 2008).

Genetic polymorphism in human populations is part of the evolutionary process that results from the interaction between the environment and the human genome. Recent changes in diet have upset this equilibrium, potentially influencing the risk of most common morbidities such as cardiovascular diseases, diabetes, and cancer. Reduction of these conditions is a major public health concern, and such a reduction could be achieved by improving our ability to detect disease predisposition early in life and by providing more personalized behavioral recommendations for successful primary prevention. In terms of cardiovascular diseases, polymorphisms at multiple genes have been associated with differential effects in terms of lipid metabolism. The integration of genetic and environmental complexity into current and future research will drive the field toward the implementation of clinical tools aimed at providing dietary advice optimized for the individual's genome (Ordovas., 2009; Engler., 2009). 
The recognition that nutrients have the ability to interact and modulate molecular mechanisms underlying an organism's physiological functions has prompted a revolution in the field of nutrition (Much et al., 2005).

For the field of nutrition, this would encompass the ongoing efforts to understand the relationships between the genome and diet, currently termed nutrigenomics and nutrigenetics (Much et al., 2005; Ommen., 2004)

\section{Nutritional genomics}

Nutrigenetics and nutrigenomics are promising multidisciplinary fields that focus on studying the interactions between nutritional factors, genetic factors and health outcomes. Their goal is to achieve more efficient individual dietary intervention strategies aimed at preventing disease, improving quality of life and achieving healthy aging (Ordovas., 2004). In contrast to most single gene disorders, chronic disorders (e. g., cardiovascular disease, cancer, diabetes) are far more complex. First, they involve multiple genes, each of which comes in more than one variation, that likely contribute in small ways to the overall condition rather than have the dramatic impact that is more typical with single gene disorders. Second, the genes are more likely to be influenced by environmental factors, which make the resulting phenotype murkier than with single-gene disorders. An individual might have gene variants that predispose to a particular chronic disorder but, depending on that individual's nutritional and other lifestyle choices, the disorder may or may not develop (DeBusk., 2008).

Nutritional genomics or nutrigenomics is the newly developing field of science that focuses on the complex interaction among genes and environmental factors, specifically bioactive components in food and how a person's diet interacts with his or her genotype to influence the balance between health and disease (DeBusk., 2009; Much et al., 2005; Fisler \& Warden., 2005).

Nutritional genomics is the umbrella term (Ryan-Harshman., 2008). There are two major subcategories of nutritional genomics: nutrigenetics and nutrigenomics (Much et al., 2005).

The creation of nutrigenomics and nutrigenetics, two fields with distinct approaches to elucidate the interaction between diet and genes but with a common ultimate goal to optimize health through the personalization of diet, provide powerful approaches to unravel the complex relationship between nutritional molecules, genetic polymorphisms, and the biological system as a whole (Much et al., 2005).

Thus, nutrition in the 21st century is poised to be an exciting and highly relevant field of research, as each new day is accompanied by advances in our understanding of how the interactions between lifestyle and genotype contribute to health and disease, taking us one step closer to achieving the highly desirable goal of personalized nutrition (Much et al., 2005).

\subsection{Nutrigenetics}

Nutrigenetics term was used first time by Dr R. O Brennan in 1975 (Farhud et al., 2010). Nutrigenetics is concerned with the effect of gene variations or gene variant or individual's genetic make-up on the organism's functional ability, specifically its ability to digest, absorb, and use food (DeBusk., 2009; Ordovas \& Corella., 2004; Lovegrove \& Gitau., 2008). Nutrigenetics embodies the science of identifying and characterizing gene 
variants associated with differential responses to nutrients or dietary pattern, functional food or supplement on a specific health outcome, and relating this variation to disease states (Much et al., 2005; Michael., 2008). The particular gene variants a person has to determine the nutritional requirements for that person and the gene-based differences in response to dietary components and developing nutraceuticals that are most compatible with health based on individual genetic makeup (DeBusk., 2009; Subbiah., 2007). Nutrigenetics will assist clinicians in identifying the optimal diet for a given individual, i. e., personalized nutrition (Much et al., 2005; Svacina., 2007; Zak \& Slaby., 2007; Gillies., 2003).

Furthermore, the concept is that if an individual is genotyped at various genes for diseaseassociated risk alleles, a genotype-based diet or nutritional supplement regimen may be useful to overcome the genetic variation and reduce risk or prevent the disease altogether (Wood., 2008; Xacur-GarcAa et al., 2008; Kussmann \& Fay., 2008).

\subsection{Nutrigenomics}

Nutrigenomics, is concerned with how bioactive components within food affect genes. The field of nutritional genomics is still evolving, and it is common to see "nutrigenomics" used as a shorthand version of "nutritional genomics ". However, keeping the concepts separate can be helpful when sorting out the underlying mechanisms involved (DeBusk., 2009). Nutrigenomics will unravel the optimal diet from within a series of nutritional alternatives, whereas nutrigenetics will help clinicians in identifying the optimal diet for a given individual, i. e., personalized nutrition (Much et al., 2005).

Although these two concepts are intimately associated, they take a fundamentally different approach to understanding the relationship between genes and diet. Despite the immediate goals differing, the long-term goal of improving health and preventing disease with nutrition requires the amalgamation of both disciplines (Much et al., 2005)

Nutrigenetics is the more familiar of the two subtypes of nutritional genomics (DeBusk., 2009) At one end of the spectrum of nutritional genomics are the highly penetrant monogenic disorders that give rise to inborn errors of metabolism such as phenylketonuria (DeBusk., 2009). More recently less penetrant, more subtle variations have been identified that also affect the gene-encoded protein's function. However, such variations do not in themselves cause disease. Instead, they alter a person's susceptibility for developing a disease. Depending on the specific gene variant, the person's likelihood of developing a disorder may be increased or reduced. These genes are the primary focus of nutritional genomics, because they are common within the global population, they affect dietary recommendations about the types and amounts of food that best fit a person, and practical interventions are possible. These interventions can potentially improve the health potential of individual people and, by extrapolation, the populations in which they live (DeBusk., 2009).

Current nutrition recommendations, directed towards populations, are based on estimated average nutrient requirements for a target population and intend to meet the needs of most individuals within that population. They also aim at preventing common diseases such as obesity, diabetes and cardiovascular disease. So diet has been reported as a major contributor to alarming prevalence of obesity (Shalileh et al, 2010). For infants with specific genetic polymorphisms, e. g. some inborn errors of metabolism such as phenylketonuria, adherence to current recommendations will cause disease symptoms and they need personalized nutrition recommendations (Hernell \& West., 2008; Farhud \& Shalileh., 2008). 
Some other monogenic polymorphisms, e. g. adult hypolactasia, are common but with varying prevalence between ethnic groups and within populations. Ages at onset as well as the degree of the resulting lactose intolerance also vary, making population-based as well as personalized recommendations difficult. The tolerable intake is best set by each individual based on symptoms. For polygenetic diseases such as celiac disease, and allergic disease, current knowledge is insufficient to suggest personalized recommendations aiming at primary prevention for all high-risk infants, although it may be justified to provide such recommendations on an individual level should the parents ask for them.

New technologies such as nutrigenetics and nutrigenomics are promising tools with which current nutrition recommendations can possibly be refined and the potential of individualized nutrition be explored. It seems likely that in the future it will be possible to offer more subgroups within a population personalized recommendations (hernell \& West., 2008).

The possibility of offering personalized nutritional advice to the individual is an attractive option for dietitians and nutrition scientists and is becoming practicable with the emergence of nutritional genomics. This developing field promises to revolutionize dietetic practice, with dietary advice prescribed according to an individual's genetic makeup to prevent, mitigate or cure chronic disease (Lovegrove \& Gitau., 2008). It has been also termed "personalized nutrition" or "individualized nutrition" (Ordovas \& Corella., 2004; PerezMartinez et al., 2011). The practical applications of this research include a new set of tools that nutrition professionals can use to identify disease susceptibilities and a growing body of knowledge that will form the basis for developing strategies for disease prevention and intervention that are specifically targeted to the underlying genetic mechanisms (DeBusk., 2008).

Nutrigenetics and personalized nutrition are components of the concept that in the future genotyping will be used as a means of defining dietary recommendations to suit the individual. Over the last two decades there has been an explosion of research in this area, with often conflicting findings reported in the literature (Rimbach \& Minihane., 2009).

According to WHO reports, diet factors influence occurrence of more than 2/3 of diseases. Most of these factors belong to the categories of nutrigenetics and nutrigenomics. In the future both, nutrigenetics and nutrigenomics, will induce many changes in preventive medicine and also in clinical medicine (Svacina., 2007).

Nutrients interact with the human genome to modulate molecular pathways that may become disrupted, resulting in an increased risk of developing various chronic diseases. Understanding how genetic variations influence nutrient digestion, absorption, transport, biotransformation, uptake and elimination will provide a more accurate measure of exposure to the bioactive food ingredients ingested. Furthermore, genetic polymorphisms in the targets of nutrient action such as receptors, enzymes or transporters could alter molecular pathways that influence the physiological response to dietary interventions. Knowledge of the genetic basis for the variability in response to these dietary factors should result in a more accurate measure of exposure of target tissues of interest to these compounds and their metabolites. Examples of how 'slow' and 'fast' metabolizers respond differently to the same dietary exposures will be discussed. Identifying relevant diet-gene interactions will benefit individuals seeking personalized dietary advice as well as improve public health recommendations by providing sound scientific evidence linking diet and health (EL-Sohemy, 2007; Amouyel., 2000). 
A really personalized diet will be a diet considering the nutritional status, the nutritional needs based on age, body composition, work and physical activities, but also considering the genotype. That is, define the "nutritional phenotype (Perez-Martinez et al., 2011; Miggiano \& De Sanctis., 2006). It is clear that integrating knowledge of gene variants into dietary recommendations for populations and individuals will increasingly play a role in nutrition counseling and policy making (DeBusk., 2008).

Nutrigenetics will provide the basis for personalized dietary recommendations based on the individual's genetic makeup. This approach has been used for decades for certain single gene diseases; such as phenylketonuria, however, the challenge is to implement a similar concept for common multi factorial disorders and to develop tools to detect genetic predisposition and to prevent common disorders decades before their manifestation. The preliminary results involving gene-diet interactions for cardiovascular diseases and cancer are promising, but mostly inconclusive. Success in this area will require the integration of different disciplines and investigators working on large population studies designed to adequately investigate gene-environment interactions (Ordovas \& Corella., 2004; Ordovas \& Mooser., 2004).

\subsection{Nutritional genomics and lipid metabolism}

From a health perspective, the major concerns regarding genes and lipid metabolism center on susceptibility to vascular disease. Genes involved with cholesterol homeostasis offer examples of how genetic variations affect lipid metabolism and, thereby, disease risk (DeBusk., 2009).

The blood lipid response to diet is influenced by polymorphisms within genes for the apolipoproteins as well as within those for enzymes, such as hepatic lipase, that are involved in lipid metabolism (fisler \& Warden., 2005).

The major focus of nutritional genomics research is on identifying (1) gene-disease associations, (2) the dietary components that influence these associations, (3) the mechanisms by which dietary components exert their effects, and (4) the genotypes that benefit most from particular dietary choices (DeBusk., 2008).

The following section takes a brief look at some of the key diet-related genes and their known variants and how these variants affect the person's response to diet. Keep in mind that chronic diseases involve complex interactions among genes and bioactive food components, and unraveling the details will require population and intervention studies large enough to have the statistical power needed to draw meaningful conclusions. Although what is known today is but the tip of the iceberg compared to what will come in the years ahead (DeBusk., 2008).

Over the last two decades there has been an explosion of research in this area, with often conflicting findings reported in the literature (Rimbach \& Minihan., 2009).

\section{Candidate gene approach}

The candidate gene approach involves the selection and study of biologically relevant genes. Genetic polymorphisms in these genes, known as Single-Nucleotide Polymorphism (SNPs), can alter susceptibility to a disease. Candidate or "susceptibility" genes should meet one or more of the following conditions: genes that are chronically activated during a disease state and have been previously demonstrated to be sensitive to dietary intervention; genes with 
functionally important variations; genes that have an important hierarchical role in biological cascades; polymorphisms that are highly prevalent in the population (usually $>10 \%$ for public health relevance); and/or genes with associated biomarkers, rendering clinical trials useful (Lovegrove \& Gitau., 2008).

Many studies have investigated this possibility and have largely focused on genes whose products affect lipoprotein metabolism, eg, apolipoproteins, enzymes, and receptors. Although there have been several reviews of such studies, many of them may have led to articles being omitted and introduced bias toward positive findings (Ordovas., 2006).

\subsection{Apolipoprotein A-I (Apo Al)}

The Apo AI gene, codes for apolipoprotein A-I, is a major structural and functional component of HDL constituting about $70 \%$ to $80 \%$ of HDL protein mass, and is the main activator of the enzyme lecithin cholesterol acyl transferase (LCAT) (Ordovas \& Corella., 2004; Lovegrove \& Gitau., 2008; Much et al., 2005; DeBusk., 2008).

Plasma HDL-cholesterol plays a protective role for CVD (Lovegrove \& Gitau., 2008; Much et al., 2005). Its gene product Apo A-I plays a central role in lipid metabolism and CVD risk. Pedigree studies have reported associations between genetic variation at the Apo A1 locus and plasma lipid and lipoprotein levels (Ordovas \& Corella., 2004). One of the variants that has been identified to be diet-related is $-75 \mathrm{G}>\mathrm{A}$, in which the typical guanine has been replaced with an adenine at position 75 within the regulatory region of the Apo A-I gene (DeBusk., 2008).

It was reported that this polymorphism was associated with Apo A-I and HDL-C concentrations, and individuals carrying the A-allele presented with the highest levels, compared with subjects homozygotes for the $G$ allele $(G / G)$ but many studies have had contradictory results (Lovegrove \& Gitau., 2008; Ordovas \& Corella., 2004).

In the context of the Framingham Heart Study, individuals with a polymorphism in the Apo A1 gene promoter region (-75 G/A) were found to respond differently to dietary PUFA (Much et al., 2005; Lovegrove \& Gitau., 2008).

The inconsistencies in reported studies outcomes are not necessarily a result of inherent differences, but are a result of a nutrient-gene interaction, i. e. a classic example of where individualized dietary advice could be important in relation to exerting a positive influence on HDL-C levels and CVD risk (Lovegrove \& Gitau., 2008; Much et al., 2005). In brief, that individuals with the A allele showed an increase in HDL levels following an increased consumption of PUFA. In contrast, those with the more common G allele showed an inverse relationship between HDL levels and PUFA consumption. This study revealed that differences in sex also mediate the response. Indeed, men did not show a relationship between HDL and PUFA consumption, irrespective of their Apo A1 polymorphism (Lovegrove \& Gitau., 2008; Much et al., 2005).

A common practice in treating dyslipidemia is to reduce the saturated fat content of the diet and increase the polyunsaturated fat content. Typically, HDL levels fall in women with the more common $\mathrm{G}$ allele as the polyunsaturated content of the diet increases, an effect counter to the desired one. These women would benefit from a fat modified diet that keeps amounts of both saturated and polyunsaturated fat low and increases amounts of monounsaturated fat. Women with the A allele, increasing polyunsaturated dietary fat leads to increased HDL levels, and the effect is "dose-dependent; so in women with the more common G allele, 
increasing dietary Polyunsaturated Fat (PUFA) levels from less than $4 \%$ of total energy to $4 \%$ to $8 \%$ to greater than $8 \%$ resulted in a corresponding decline in HDL levels as PUFA levels increase. However, in women with the A allele, increasing PUFA concentrations $(>8 \%$ of energy derived from PUFA) increased HDL levels and the increase is more dramatic in the presence of two copies of the A alleles than it is with just one. For these women, a diet low in saturated fat, moderate in polyunsaturated fat ( $8 \%$ or greater of total calories), and supplying the rest in monounsaturated fat has the greatest benefit in raising HDL levels. Clearly, whether a person has the $-75 \mathrm{G}>\mathrm{A} A$ po $A I$ variant, and how many copies are present, will affect any therapeutic intervention developed to correct dyslipidemia (DeBusk., 2009; Much et al., 2005; Debra., 2008).

Juo et al (Hank Juo., 1999) used a meta analysis approach to show the lack of consistency between the less common A-allele and higher HDL-cholesterol concentrations. In view of the significant gene-diet interaction observed for those intervention studies, they examined whether these results could be extrapolated to a free living population, consisting of about 1600 Framingham Offspring Study participants (Ordovas et al., 2002). The results from the straightforward association between genotype and phenotype were disappointing and suggested that the G/A polymorphism was not associated with HDLcholesterol, Apo A-I concentrations, nor with any other anthropometrical or plasma lipid variable examined. To examine the potential modifying effect of dietary fat on these associations, they fitted multivariate linear regression models, including interaction terms for fat intake [total, Saturated Fatty Acid (SFA), Monounsaturated Fat (MUFA), and PUFA fat]. No significant interactions were observed between the G/A polymorphism, total, SFA, and MUFA fat intakes. However, in women, HDL-cholesterol concentrations were associated with a significant interaction between PUFA intake and Apo A1 genotype $(p=0.005)$. Using PUFA as a dichotomous variable, their data show that $G / G$ women consuming $<6 \%$ PUFA/day had higher HDL-cholesterol $(1.48 \pm 0.40 \mathrm{~m} \mathrm{~mol} / \mathrm{L})$ than Acarriers (1. $43 \pm 0.40 \mathrm{~m} \mathrm{~mol} / \mathrm{L})$. Conversely, when consuming $\geq 6 \% \mathrm{PUFA} /$ day, G/G had lower HDL-cholesterol concentrations $(1.44 \pm 0.39 \mathrm{~m} \mathrm{~mol} / \mathrm{L})$ than A-carriers $(1.49 \pm 0.39$ $\mathrm{m} \mathrm{mol} / \mathrm{L})$. In men, the situation was more complex because the effects were observed using three-way interactions, including smoking and alcohol consumption, in the analyzes (Ordovas \& Corella., 2004).

The most evident application of these results may be to help us make more efficacious dietary recommendations based on genetic profile. It is clear that subjects with the A-allele at this Apo A1-75 (G/A) polymorphism will benefit from diets containing a high percentage (it is important to underscore that we are talking about percent in the diet and not about total amounts) of PUFA (i. e., vegetable oils, fish, nuts, and so on). According to their data, this should result in higher HDL-cholesterol concentrations, which in turn should lower CVD risk. These findings suggest that the expression of the Apo A1 gene may be regulated by PUFA (Ordovas \& Corella., 2004).

On the other hand, of 13 reports, 5 found that the presence of the Apo A-I-75 (G/A) A allele instead of the common $G$ allele resulted in greater LDL-cholesterol responses to changes in dietary. In addition, significant interactions between the $G / A$ genotype and diet were found for changes in total and LDL cholesterol when subjects changed from a low-fat diet to a diet high in MUFAs. No significant interactions between diet and other polymorphisms in the Apo A-I gene were shown (Ordovas \& Corella., 2004). 


\subsection{Apolipoprotein A-IV (APOA4)}

Apo A-IV is a 46-Kd plasma glycoprotein that is synthesized by intestinal enterocytes during lipid absorption and is incorporated into nascent chylomicrons. Apo A-IV enters circulation on lymph chylomicrons, but then dissociates from their surface and circulates primarily as a lipid-free protein. Several genetically determined isoforms of Apo A-IV have been detected; amino acid positions 360 and 347 of the mature protein are the most common. The polymorphism at position 360 is due to a CAG $\rightarrow$ CAT substitution at codon 360 in the Apo A4 gene and encodes a Q360H (Gln $\rightarrow$ His) substitution in the carboxyl terminus, and produces an isoform, originally known as Apo A-IV-2, one charge unit more basic than the common isoform, Apo A-IV-. In some population studies the Apo A-IV-2 allele is associated with higher levels of HDL-cholesterol and or Apo A-I and/or lower triglyceride (TG) levels, as well as lower LDL-cholesterol, lower Lp (a), and higher fasting glucose and insulin levels, but no associations have been observed in other studies (Ordovas \& Corella., 2004).

The other common mutation (Thr347 $\rightarrow$ Ser) is due to an ACT $\rightarrow$ TCT substitution at codon 347 in the human Apo A-IV gene, it is found within subjects with the apoA-IV-1 isoform. Several population studies note that carriers of the $347 \mathrm{~S}$ allele have lower plasma, total, LDL-cholesterol, Apo B levels, and Lp (a) levels, than 347T/T homozygotes. The results of many reports showing that male carriers of the less common allele at the Gln $360 \mathrm{His}$ polymorphism were less responsive to changes in dietary fat and cholesterol or cholesterol alone (Ordovas \& Corella., 2004).

Several studies have focused on the interaction between the Apo A4 locus and dietary factors, both in the fasting and postprandial states. Similar to the findings for other genes, the data are conflicting when it comes to the effect of Apo A-IV polymorphisms on the LDL response to dietary cholesterol. However, according to Weinberg (Weinberg., 2002), the results from different studies can be partially reconciled if one assumes that the dietary fatty acid effects dominate over the allele effects. Therefore, if dietary cholesterol intake is the principal variable, and total fat intake is moderate and constant, $\mathrm{Q} / \mathrm{H}$ subjects display an attenuated response of LDL-cholesterol. However, when dietary cholesterol intake is changed in the setting of a higher baseline dietary fat intake or with a change in fat saturation, the fatty acid effects on LDL levels predominate and overrule the allele effect. The impact of the Q360H polymorphism on cholesterol absorption may be greater on a high PUFAs intake. However, dietary PUFA counteract the effect of dietary cholesterol on the expression of hepatic LDL receptors. Thus, the final effect of Apo AIV alleles on the LDL response to dietary cholesterol may be determined by the relative amounts of cholesterol, saturated fatty acids (SAFAs), and PUFAs in the diet (winberg et al., 2000; Weggemans., 2000; Lopez-Miranda., 1998; Ordovas \& Corella., 2004; Hockey., 2001).

There is more consistency and probably less complexity regarding the impact of Apo A-IV polymorphisms on HDL-cholesterol: When total fat intake is raised or lowered, $\mathrm{Q} / \mathrm{H}$ subjects have an exaggerated, and Threonine /Serine (T/S) subjects an attenuated, response in plasma HDL levels. It has been suggested, and Weinberg demonstrated, that a highPUFA intake may amplify this effect (Ordovas \& Corella., 2004; Winberg et al., 2000).

Given the relationships between plasma TG and plasma HDL-cholesterol levels, it is possible that the response of plasma HDL-cholesterol levels to changes in dietary fat is mediated by Apo A-IV allele effects on postprandial triglyceride-rich lipoprotein metabolism. These studies clearly illustrate the extreme complexity associated with the 
interpretation of results from studies involving gene-diet interactions (Ordovas \& Corella., 2004).

The presence of serine instead of threonine at position 347 in the Apo A-IV gene was associated with increased total and LDL-cholesterol responsiveness when subjects switched from a high-SFA diet to a National Cholesterol Education Program Step I diet. When the same subjects changed from the National Cholesterol Education Program Step I diet to a high-MUFA diet, subjects with the Thr /Thr genotype had a 1\% decrease in total cholesterol concentrations, whereas subjects with the Ser allele had a 5\% increase in total cholesterol concentrations. When the Thr347Ser and the Apo A-I-75 (G/A) genotypes were combined, carriers of the $A$ and Ser alleles showed greater LDL-cholesterol responses to changes in dietary fat. However, Carmena-Ramon et al (Carmena-Ramon et al., 1998) investigated both the Gln360His and Thr347Ser polymorphisms and found no gene-diet or haplotype-diet interactions (Masson et al., 2003). The evidence that exists for an interaction between diet and the Apo A-IV glutamine-histidine mutation at position 360 (Gln360His) suggests that Gln / Gln subjects show significantly greater total and LDL-cholesterol responses and that $\mathrm{Gln} / \mathrm{His}$ subjects show greater HDL-cholesterol responses to changes in dietary fat, cholesterol, or both. Although Wallace et al found no significant differences in LDLcholesterol responses between genotypes, dense LDL cholesterol decreased more in subjects carrying the His allele when polyunsaturated fatty acids (PUFAs) replaced SFAs in the diet (Wallace et al, 2000). In the same study, there was a significant difference in HDL-cholesterol responses between genotype groups such that concentrations decreased in Gln /Gln subjects and increased in Gln /His subjects (Masson et al., 2003).

\subsection{Apolipoprotein B (Apo B)}

Apolipoprotein $B$ is the main protein component of low-density-lipoprotein (LDL) and contains several domains. The human Apo B is $43 \mathrm{~kb}$ in length with $81 \mathrm{bp}$ signal sequence. Numerous polymorphisms have been identified on this gene (Heilbronn et al., 2000).

The evidence for an interaction between the XbaI polymorphism and diet is inconsistent. In 2 studies, $X-X$ - subjects showed greater LDL-cholesterol responses, whereas Tikkanen et al, found that subjects carrying the $X^{+}$allele had greater total, LDL-, and HDL-cholesterol responses. However in, analysis of these data, the XbaI polymorphism only explained a significant proportion of variance of the change in HDL cholesterol (Tikkanen et al., 1995). In one research they found no significant effect on LDL-cholesterol responsiveness, although $X-X$-subjects showed the greatest $\mathrm{HDL}_{2^{-}}$and VLDL-cholesterol responses. Finally, in another study researchers studied the effect of the XbaI polymorphism in subjects with the common Apo E3/3 genotype and found that $X-X$ - subjects showed the greatest triacylglycerol response. Rantala et al conducted a meta-analysis of all published dietary trials. In their analysis of 8 studies, $X-X+$ subjects had greater LDL responses than did $X+X+$ subjects and no significant differences in the responses of total or HDL cholesterol or triacylglycerol were found between genotypes (Masson et al., 2003; Rantala., 2000). Two of 7 intervention studies found that the EcoRI $R$ - allele was associated with significantly greater total and LDL-cholesterol responses to changes in dietary fat and cholesterol. Only one study found an interaction between the MspI polymorphism and response to diet. Ten intervention studies found no significant effects of the Apo B signal peptide insertion/deletion $(I / D)$ polymorphism on dietary responsiveness; however, 2 studies reported a significantly greater responsiveness in subjects homozygous for the $I$ allele. In a 
study, 43 men and women were observed to compare the effects of insoluble and soluble fiber on plasma lipids. Their statistical model identified gene-diet interactions. However, they did not look specifically at differences between genotype groups. It was found that $D / D$ subjects had similar decreases in HDL cholesterol after consumption of the insoluble- and soluble-fiber diets. However, I/I subjects had larger HDL-cholesterol decreases with the soluble-fiber diet, whereas $I / D$ subjects had larger HDL-cholesterol decreases with the insoluble-fiber diet. The gene-diet interaction was significant $(P=0.021)$ (Masson et al., 2003; Rantala., 2000).

In response, low-fat, low-cholesterol diet, I/I subjects showed the greatest decrease in HDL2. In addition, $I / I$ and $I / D$ subjects showed increased VLDL-cholesterol and decreased LDLcholesterol concentrations, whereas $D / D$ subjects showed decreased VLDL-cholesterol and increased LDL-cholesterol concentrations. The I/D polymorphism showed no significant effect on the responsiveness of total, LDL, or HDL cholesterol or triacylglycerol in a metaanalysis of 7 studies (Masson et al., 2003).

\subsection{Apolipoprotein E (APO E)}

Apo E gene variants have implications for nutrition therapy related to preventing and treating CVD and the responses to dietary fat, soluble fiber, and alcohol. The impact of Apo E genotype on individual variability in its LDL cholesterol response to diet interventions and CVD risk has been extensively investigated over the past 30 years. Apo E contains 299 amino acids, considering Apo E's key role in lipoprotein metabolism, being involved in chylomicron metabolism, very low-density lipoprotein synthesis and secretion, and in the cellular removal of lipoprotein remnants from the circulation. Apo E serves as a ligand for multiple lipoprotein receptors. This gene locus is polymorphic, with 84 gene variants being characterized to date. The prevalence of this SNP varies in different populations (Lovegrove \& Gitau., 2008; Rubin \& Berglund., 2002; Ordovas \& Corella., 2004; DeBusk., 2009).

Apo $\mathrm{E}$ is present in a subfraction of lipoprotein (a). The receptor-binding properties reside in the N-terminal part of Apo E, whereas the lipid-binding domain resides in the C-terminal portion. It was recognized that Apo E was present as three different Apo E isoforms (E2, E3, and E4), coded by three different alleles (e2, e3, and e4), resulting in six homo and heterozygous genotypes (e2/e2, e2/e3, e2/e4, e3/e3, e3/e4, e4/e4). Apo E2 differs from the wild type, Apo E3, by a substitution of arginine for cysteine at amino acid 158, and Apo E4 differs from Apo E3 by a substitution of cysteine for arginine at amino acid 112. In addition, several other genetic variants have been described at the Apo E locus (Rubin \& Berglund., 2002; Lovegrove \& Gitau., 2008; Masson et al., 2003; Farhud et al., 2010).

Persons with E4 variant respond to a high-fat diet negatively with an increased risk for coronary heart disease (CHD). In these individuals, low-fat diet should be useful (Farhud et al., 2010; Sheweta et al., 2011).

Population studies show that plasma cholesterol, LDL cholesterol, and Apo B levels are highest in subjects carrying the Apo E4, intermediate in those with the Apo E3, and lowest in those with the Apo E2 isoform. An initial observation was that the association of the Apo E4 isoform with elevated serum cholesterol levels was greater in populations consuming diets rich in saturated fat and cholesterol than in other populations (Ordovas \& Corella., 2004).

Corella and Ordovas reviewed the numerous studies that have investigated the diet-gene interaction for Apo E variants. People with at least one E4 allele have the highest basal levels 
of various lipids and show the greatest lipid-lowering response to a low-fat diet. Taking into account which Apo E alleles a person has is helpful in developing diet and lifestyle interventions for improving serum lipid levels (DeBusk., 2009).

In 46 studies that examined the Apo E locus and alterations in dietary fat content, significantly different responses in total and LDL cholesterol by Apo E genotype were reported in 8 and 11 studies, respectively, with the Apo E4 individuals generally being the most responsive (Lovegrove \& Gitau., 2008; Masson et al., 2003).

Note that despite the numerous studies examining the relation between Apo E genetic variability and LDL-cholesterol response to diet intervention, there is considerable inconsistency regarding the magnitude and significance of the reported associations, and this locus continues to be the subject of intense research (Ordovas \& Corella., 2004).

In a study, there are 29 intervention studies that examine Apo E-diet interactions. A total of 3224 subjects participated in these studies, ranging from 16 to 420 subjects per study. Of the 29 studies, 12 demonstrated no significant Apo E-diet interactions, 15 reported significant interactions (E4 was usually associated with increased dietary response), and 2 were undefined. Using the same available literature, but different selection criteria, Masson, reviewed 62 dietary intervention periods, including 3223 subject-by-diet interventions (Masson et al., 2003). Again, the range of the studies varied between 8 and 210 subjects per dietary intervention. According to this review, 42 of the diet interventions did not demonstrate significant Apo E-diet interactions, and only 19 provided evidence for significant interactions, clearly demonstrating the diversity of the results presented in the original papers as well as those obtained from review papers (Ordovas \& Corella., 2004; Masson et al., 2003).

The heterogeneous response to changes in dietary fat may be attributed to a number of factors including age, gender, baseline LDL-C levels, disease status and drug use (Lovegrove \& Gitau., 2008).

One difference between the negative studies and those reporting significant Apo E gene-diet interactions relates to the baseline lipid levels of the subjects. Studies reporting significant associations often included subjects who were moderately hypercholesterolemic and/or had significant differences in base total cholesterol and LDL-cholesterol among the Apo E genotype groups. This suggests that the significant gene-diet interaction is apparent only in subjects susceptible to hypercholesterolemia. Concerning differences in dietary interventions, there were significant interactions in studies in which total dietary fat and cholesterol were modified. Several mechanisms are proposed to explain these Apo E-related differences in individual response to dietary therapy. Some studies show that intestinal cholesterol absorption is related to Apo E phenotype, with Apo E4 carriers absorbing more cholesterol than non-Apo E4 carriers. Other mechanisms such as different distribution of Apo E on the lipoprotein fractions, LDL Apo B production, bile acid, and cholesterol synthesis, and postprandial lipoprotein clearance may also be involved (Ordovas \& Corella., 2004).

On the other hand although the obvious dietary factors implicated in gene-diet interactions affecting plasma lipid levels are dietary fats and cholesterol, other dietary components have revealed significant interactions. This is the case for alcohol intake.

Although the raising effect of alcohol consumption on high-density lipoprotein (HDL)cholesterol levels is well established, the effect on LDL-cholesterol is still unclear. It is possible that the reported variability will be due to interactions between genetic factors and 
alcohol consumption. Using cross-sectional analysis, researcher examined whether variation at the Apo E locus modulates the association between alcohol consumption and LDLcholesterol levels in a healthy population based sample of 1014 male and 1133 female participants in the Framingham Offspring Study (Corella et al., 2001). In male nondrinkers, LDL-cholesterol levels were not different across Apo E groups; however, in male drinkers, there were differences in LDL-cholesterol, with Apo E2 subjects displaying the lowest levels. When LDL cholesterol levels were compared among the Apo E subgroups by drinking status, LDL-cholesterol levels in Apo E2 male drinkers were lower than in Apo E2 non drinkers. Conversely, in Apo E4 males, LDL-cholesterol was higher in drinkers than in nondrinkers. This Apo E-alcohol interaction remained significant after controlling for age, BMI, smoking, fat, and energy intake. In women, the expected effect of Apo E alleles on LDL-cholesterol levels was present in both drinkers and nondrinkers. Multiple linear regression models showed a negative association between alcohol and LDL-cholesterol levels in Apo E2 men, with alcohol intake a continuous variable. Conversely, in Apo E4 men, this association was positive. There were no statistically significant associations in either Apo E3 men or in women. These data suggest that in men, variability at the Apo E locus partially modulates the effects of consuming alcoholic beverages on LDL-cholesterol levels (Ordovas \& Corella., 2004).

The effect of alcohol was also investigated in the Copenhagen City Heart Study (FrikkeSchmidt., 2000). In that study, there was an interaction between alcohol and Apo E among women, in which higher triglyceride levels were associated with both the E2 and E4 alleles among women who regularly consumed alcohol. For men, increased triglyceride levels among E2 and E4 carriers were seen across the entire alcohol distribution spectrum, perhaps because of some degree of alcohol consumption among all men. Overall, the results suggested that metabolic stresses, such as the postprandial situation or alcohol consumption, might contribute to uncover underlying differences between Apo E genotypes in cholesterol, triglyceride or lipoprotein metabolism (Frikke-Schmidt., 2000).

The effect of the Apo E gene on lipoproteins may differ with age. In elderly individuals as well as in children, there is less difference in LDL cholesterol levels in individuals carrying the E4 allele versus non-E4 carriers. Interestingly, in both of these age groups, the presence of the Apo E2 allele was associated with lower LDL cholesterol levels. An age-dependent variation between Apo E and plasma lipids was also seen by Jarvik et al (Jarvik et al, 1997). By longitudinally following male Caucasian twins, the authors demonstrated that whereas E4 carriers initially had higher triglyceride and cholesterol levels compared with E3 homozygotes, this difference disappeared over an 18-year period (Rubin \& Berglund., 2002). A sex-specific association between Apo E2 and HDL cholesterol levels has been described in Turkish individuals. In Turkish women, but not men, the frequency of the Apo E2 allele increased almost six fold from the lowest to the highest HDL cholesterol tertiary (Rubin \& Berglund., 2002). The available information show, significant diet-Apo E gene interactions occurred in male-only studies. In studies including men and women, significant effects were noted only in men, suggesting a significant gene-sex interaction (Ordovas \& Corella., 2004). As pointed out above, in studies in which an Apo E gene nutrient interaction was found, it was generally more common among men than women, suggesting a modulation by sex. Interestingly, in the study by Mahley et al, on HDL levels in Turkish individuals, the authors suggested that the association of Apo E2 with higher HDL cholesterol levels found in women but not in men may be caused by a sex difference in hepatic lipase. Among 
women, a lower hepatic lipase activity might allow the detection of the modulating effect of Apo E genotypes, whereas this effect might be overwhelmed by a higher enzyme activity in men (Mahley et al., 2000). This is an analogous situation to the suggestion above that differences in susceptibility might be uncovered by a metabolic challenge (Rubin \& Berglund., 2002).

Other causes for the observed differences between studies may be the presence of confounders, the type of dietary intervention used, the population studied and, importantly, the number of subjects in the respective studies. A small number of subjects limits the possibility of detecting differences, or could alternatively lead to spurious associations. Although the number of studies addressing the gene nutrient interaction for Apo $E$ is growing, in most studies so far this has been a secondary endpoint, usually analyzed post hoc. Perhaps the most likely possibility is that a number of dietary interventions will elicit variable responses across Apo E genotypes, but that the ability to detect such differences will depend on the strength and type of intervention as well as on specific recipient factors (type of population, presence of hyperlipidemia, etc. ). In the end, however, our ability to confirm or refute the presence of Apo E gene nutrient interactions as well as to understand their metabolic basis fully will require larger and more detailed studies (Rubin \& Berglund., 2002).

Inconsistency in nutrient-gene interactions in relation to Apo E polymorphisms may be a result, in part, of retrospective genotyping of small study cohorts, for which the genotypediet-LDL-C interactions were not the primary outcome. This factor has resulted in the under-representation of the less-frequent genotypes and, although trends may have been evident, many of the studies were clearly under-powered to detect significant genotypetreatment effects. The prospective genotyping of larger study cohorts has been used as an alternative approach to increase statistical power (Lovegrove \& Gitau., 2008).

However, recent evidence strongly suggests that variations in a number of key genes may also be important, including common variants of the Apo E gene. The most convincing evidence to date for genotypic effects on dietary response comes from the extensively studied Apo E gene variant (Lovegrove \& Gitau., 2008).

A metaanalysis has been published recently that summarizes the overall findings from studies using a variety of end-point measures. A mean $40-50 \%$ increase in CHD risk was observed in E4 carriers (overall OR 1. 42) relative to the wild-type E3/E3 genotype, with no apparent differences for either the E2 and E3 subgroups (OR 0. 98). Although a causal mechanism to link E4 with increased CHD risk has not been fully elucidated, the association has been ascribed to a higher concentration of LDL-C. This higher LDL-C is believed to arise from the Apo E4 isoform having a relatively higher affinity for its membrane (LDL/chylomicron remnant) receptor and feedback inhibition of receptor activity in E4 carriers Other mechanisms relating to reduced antioxidant status may also be operative (Lovegrove \& Gitau., 2008).

There is the large variation that is observed in the concentration of serum LDL-cholesterol (LDL-C) in response to fish oil supplementation. The cardioprotective effects of the fatty acids in fish oil include eicosapantanoiec acid (EPA) and docosahesanoiec acid (DHA) are well recognized. However, a potentially deleterious increase in LDL-C (5-10\%) has been consistently reported after moderate to high doses of fish oil (>2 g EPA+ DHA/d). These data showed the DHA rather than the EPA in fish oils that is responsible for the LDL-C raising effects in E4 individuals (Lovegrove \& Gitau., 2008). 
In contrast, those with one or more E4 alleles have the highest serum total cholesterol, LDL-, and Apo B levels, the lowest HDL-C levels, and have elevated fasting and postprandial triglyceride levels. They respond best to a low-fat diet but are the least responsive to soluble fiber for lowering serum lipids or to exercise for increasing HDL levels. Fish oil supplementation in these people increases total cholesterol and reduces HDL. Whether a person has the $U$ allele or the E4 allele appears to make a difference in the diet and lifestyle recommendations that would be appropriate for improving vascular health (DeBusk., 2009). Two prospectively genotyped studies designed to test the hypothesis that Apo E polymorphism has a significant effect on the LDL-C response to EPA and DHA have recently been completed (Lovegrove \& Gitau., 2008).

Overall, the triglyceride response to the fat load was lower during fish oil supplementation, and interestingly the decrease in the incremental area under the curve for triglyceride levels was significantly higher for E2 carriers compared with E3 homozygotes and E4 carriers (Rubin \& Berglund., 2002).

Although a number of previous studies have observed effects of Apo E genotype in response to dietary total fat and saturated fatty acid (SFA) manipulation, only one study to date has examined the Apo E genotype-dietary fat-LDL-C association using prospective recruitment by genotype. A study reported a significant effect of Apo E genotype on the plasma lipid response to a low fat diet, with a 5\%,13\% and $16 \%$ reduction in LDL-C in $\mathrm{E} 3 / \mathrm{E} 3, \mathrm{E} 3 / \mathrm{E} 4$ and E4/E4 males, respectively. Other studies have examined the association between Apo E genotype and fish oil (EPA/DHA) on LDL-C responses. In a retrospectively genotyped study it was observed that a mean increase of 7. 1\% in LDL-C for the group as a whole was solely attributable to a $16 \%$ rise in LDL-C in the Apo E4 participants, and it was speculated that Apo E genotype may, in part, predict the blood lipid response to fish oil intervention. Variable effects of EPA and DHA on LDL-C have been reported previously (Kobayashi et al., 2001; Lovegrove \& Gitau., 2008).

The ApoE gene locus accounts for approximately $7 \%$ of the population variance in total and LDL cholesterol levels; in general, E4 carriers have higher and E2 carriers have lower LDL cholesterol levels. It has also been suggested that Apo E variations impact triglyceride levels, as higher triglyceride levels have been reported for both E4 and E2 carriers compared with E3 homozygotes (Rubin \& Berglund, 2002).

In a recent study of more than 9000 individuals from the Copenhagen City Heart Study, Frikke-Schmidt and colleagues demonstrated that the association between the Apo E locus and cholesterol or plasma Apo B levels was invariant, i. e. present in most contexts (e. g. present in both men and women), whereas associations between Apo E and other lipoproteins such as triglycerides, Apo A-I, HDL cholesterol and lipoprotein (a) were found to be context dependent (Frikke-Schmidt., 2000). As the associations of Apo E with Apo B remained significant when adjusting for cholesterol but not the other way around, this suggested that Apo B is the factor primarily associated with Apo E genotype. It should be pointed out, however, that in their study triglyceride levels represented nonfasting conditions, and LDL cholesterol was not included in the analysis (Rubin \& Berglund., 2002). Furthermore, the Apo E2 allele was more common in individuals with high LpA-I levels, i. e. HDL with Apo AI but not Apo A-II. This HDL subfraction generally corresponds to the larger HDL2 subpopulation, which interestingly, in a study by Isasi et al, was associated with Apo E2 in children (Isasi et al., 2000). In view of their results, Mahley et al, suggest that HDL containing Apo E2 might be a poorer substrate for hepatic lipase compared with HDL 
with Apo E3 or E4, leading to an accumulation of HDL in plasma. In addition, there might be a difference in the clearing mechanisms between HDL containing Apo E2 compared with Apo E3 or E4 (Mahley et al., 2000).

This mixed pattern was recently addressed by Weggemans et al, who performed a metaanalysis of 26 controlled clinical diet trials conducted. The effect of Apo E genotypes on response to dietary change in 395 healthy subjects, well balanced for sex, was evaluated. The authors pooled data in the response of LDL and HDL cholesterol from four types of trials; replacement of cis-unsaturated fat for saturated fat ( $\mathrm{n}=7$ studies), replacement of cisunsaturated fat for trans unsaturated fat $(n=2)$, changes in dietary cholesterol $(n=8)$ and changes in coffee diterpenes $(n=9)$. Overall, there were small, non-significant differences between Apo E genotypes in the response of LDL cholesterol, and results were unchanged after adjusting for age, sex and body mass index. For HDL cholesterol, a sex difference was noted, as the response to trans fat and cholesterol differed across Apo E genotypes in men but not in women (Weggemans et al., 2001).

Appropriately, the authors caution against the over interpretation of this result because of chance associations (Rubin \& Berglund., 2002).

Friedlander et al, compared plasma levels across Apo E genotypes in response to two diets, a high saturated fat/high cholesterol and a low saturated fat/low cholesterol diet, in 214 free-living individuals in two kibbutz settlements in Israel. Although the baseline total and LDL cholesterol levels were higher among E4 carriers and lower among E2 carriers compared with E3 homozygotes, the plasma lipid response to the diet intervention did not differ across Apo E genotypes (Friedlander et al., 2000).

Loktionov et al, investigated 132 free-living healthy individuals participating in the European Prospective Investigation of Cancer study, a cohort study with approximately 25 000 subjects. The reported subgroup was part of a quality control study on the dietary methods used. In the 132 subjects, serum cholesterol levels correlated with the intake of total and saturated fat. For LDL cholesterol, a significant correlation with relative saturated fat intake was seen only for Apo E 4/3, and not for Apo E3/3 or 3/2 (Loktionov et al., 2000).

In another recent study, researchers analyzed lipid levels in relation to Apo E genotypes in 420 randomly selected free-living Costa Rican individuals consuming a low fat intake (53\% of energy). In accordance with most previous studies, E2 carriers had lower, and E4 carriers higher LDL cholesterol and Apo B levels compared with E3 homozygotes. The population was dichotomized in two groups depending on the intake of saturated fat. High saturated fat intake (mean intake 13. 5\% of energy) was associated with increased VLDL cholesterol, decreased HDL cholesterol and smaller LDL sizes in Apo E2 carriers, whereas the opposite was found for Apo E4 carriers. Effects on LDL size had previously been noted by Dreon et al, in which a more pronounced decrease in large, buoyant LDL particles during reduced fat intake was seen for Apo E4 carriers (Dreon et al, 1995). The findings of Campos et al suggested, as pointed out by the authors, that in E2 carriers, a high saturated fat intake may result in increased VLDL production and delayed clearance. Such a metabolic challenge might thus unmask a relative susceptibility in E2 carriers (Campos et al, 2001).

Finally, the study on plasma lipid response to dietary fat and carbohydrate in men and women with coronary heart disease provided further support for the association of triglyceride metabolism with Apo E2. Overall, E2 carriers had lower LDL cholesterol as well as a tendency to higher triglyceride levels than E3 and E4 carriers. In addition, there was a positive association between dietary sucrose $(6 \pm 7 \%$ of the total energy intake) and plasma triglyceride levels among E2 carriers (Rubin \& Berglund., 2002). 
Postprandial studies as Apo E have important functions in chylomicron remnant metabolism, there has been substantial interest in the role of Apo E genotypes in the postprandial setting. Furthermore, a postprandial challenge could serve as a tool to uncover more precisely the differences between different Apo E alleles (Rubin \& Berglund., 2002).

In a study of normolipidemic adults by Rubin \& Berglund, the Apo E2 allele was associated with an increased postprandial triglyceride response. A similar response has also been demonstrated in other studies. Regarding the Apo E4 allele, more controversial results have been obtained (Rubin \& Berglund., 2002).

However, although such studies are compatible with a faster clearance of VLDL and chylomicron remnants in E4 compared with E3 carriers, the meta-analysis showed higher triglyceride and lower HDL cholesterol levels among E4/3 individuals compared with E3 homozygotes. This would perhaps suggest an impaired postprandial clearance among E4 carriers (Rubin \& Berglund., 2002). In support of this, another study found an impaired clearance of chylomicron and VLDL remnants in normolipidemic male E4 carriers compared with E3/3. Furthermore, several recent studies have also reported an increased postprandial triglyceride excursion in E4 carriers. In children, they did not observe any difference in triglyceride or retinyl palmitate response between E3/3 and E4 carriers, although a nonsignificant trend towards higher baseline triglyceride levels as well as higher triglyceride and retinyl palmitate levels $3 \mathrm{~h}$ postprandially among E4 carriers was seen (Couch et al., 2000). Another research found no significant effects of the Apo E4 allele on the postprandial triglyceride response after adjusting for baseline triglyceride levels, although a delayed retinyl palmitate clearance in E2 carriers was observed (Rubin \& Berglund., 2002). In a recent study by Kobayashi et al, individuals with the E3/3 and E3/4 genotypes were matched for intra-abdominal visceral fat accumulation. Postprandial triglyceride levels did not differ between the two genotypes when adjusting for baseline levels, whereas retinyl palmitate levels among lipoproteins with Sf 5400 were higher among male E3/4 subjects, indicating a slower remnant clearance. As pointed out by the authors, there were fewer women in the study, which might contribute to the non-significant finding in this sex group (Kobayashi et al., 2001).

In a study, the researchers investigated postprandial fat load tolerance in 55 healthy volunteers with an atherogenic lipid profile, defined as triglyceride levels of $1.5 \pm 4 \mathrm{~m} \mathrm{M}$, cholesterol $5 \pm 8 \mathrm{~m} \mathrm{M}$ and HDL cholesterol less than $1.1 \mathrm{~m} \mathrm{M}$, as part of a double-blind placebo-controlled crossover study with the consumption of either $6 \mathrm{~g}$ of fish oil or $6 \mathrm{~g}$ of olive oil supplements for 6 weeks. At the end of each period, a postprandial study was carried out. The difference in LDL cholesterol levels among Apo E genotypes is associated with differences in LDL receptor activity, with Apo E2 carriers having higher and Apo E4 carriers lower activity compared with Apo E3 homozygotes. Conditions with increased stress of this system, such as the increased intake of cholesterol and saturated fat, could therefore result in a variable response in LDL cholesterol levels across Apo E genotypes. In addition, E2 carriers may have decreased lipolytic function with an inhibition of the conversion of VLDL to LDL, as well as a compromised clearance system for triglyceride-rich lipoproteins. Therefore, even a modestly increased VLDL production in response to increased precursor availability might result in differences in plasma triglyceride levels across Apo E genotypes (Rubin \& Berglund., 2002).

Although the results from postprandial studies are generally in agreement with the established metabolic differences between Apo E2 and E3, it is currently more difficult to 
explain the reduced postprandial clearance in E4 carriers. However, it is possible that a differential distribution of the varying Apo E isoforms over different lipoprotein fractions, as well as variations in Apo E levels, could play a role. In addition, a lower LDL receptor activity in E4 carriers may contribute to a decreased postprandial clearance (Rubin \& Berglund., 2002).

How could we reconcile these varying results? Even if most studies have established associations between Apo $\mathrm{E}$ and baseline lipoprotein levels, the absolute differences between the Apo E genotypes are relatively modest. It might thus be expected that intergenotype differences in response to nutrient variations may generally be even smaller in magnitude, and thus more difficult to detect, although they might be enhanced by metabolic challenges affecting the synthetic or clearance systems in lipoprotein metabolism described above. Examples of such metabolic stresses in which Apo E gene nutrient interactions may be more readily detectable may be hyperlipidemia, an increased intake of saturated fat or cholesterol, the postprandial state, or alcohol intake. In agreement with this, studies indicating Apo E gene nutrient interactions have been more common in hyperlipidemic settings, whereas it has been more difficult to detect differences across Apo E genotypes in normolipidemic individuals or populations. However, Apo E gene \pm nutrient interaction has not been seen in all hyperlipidemic states. In familial heperlipidemia heterozygotes, no difference in plasma lipid response to a step 1 diet was seen across Apo E genotypes, indicating that the modulating effects of Apo $\mathrm{E}$ may be overwhelmed by other genetic defects, such as LDL receptor deficiency (Rubin \& Berglund., 2002).

In conclusion, Apo E has important functions in lipoprotein metabolism and the Apo E polymorphism is associated with plasma lipoprotein levels. Although a large number of studies have addressed whether there is an interaction between Apo E genotypes and diet in affecting plasma lipid levels, this issue is presently unresolved. Most studies to date have involved a small number of subjects, analyzed the Apo E polymorphism post hoc, or included populations in which the effects might be modest, making discrepancies difficult to detect. Studies conducted with conditions representing a metabolic challenge have generally been more successful in finding differential effects across Apo E genotypes, and such studies may be helpful in the future to clarify Apo E gene nutrient relationships. The mixed results obtained indicate that, at present, it is premature to suggest the use of genotyping of Apo $\mathrm{E}$ in the design of therapeutic diet interventions (Rubin \& Berglund., 2002).

All studies have demonstrated a strong association between plasma cholesterol and Apo E phenotypes in the following order: E4/E4 > E4/E3 > E3/E3 > E3/E2. It has been thought possible that the Apo E gene might be involved in the modulation of dietary plasma cholesterol responses, perhaps explaining the differences in cholesterol concentrations. Some dietary intervention studies have suggested that Apo E4 individuals react to dietary change with exaggerated cholesterol responses. In one study, Apo E4/E4 individuals responded by increased cholesterol reductions during low fat intake, and by increased cholesterol elevations during a switchback to high fat diet. Plausible mechanisms have been postulated which could explain such differences. However, other studies have reported no differences in plasma lipid responses among Apo E phenotypes. The studies cannot be directly compared because of different designs and study populations with differing Apo E allele frequencies (Tikkanen., 1995).

Although Tikkanen et al, found that subjects with the E4/4 phenotype showed the greatest total and LDL-cholesterol responses to dietary change (Tikkanen et al., 1995) Xu et al 
analyzed the same data and concluded that the Apo E polymorphism did not explain a significant proportion of the variation of the response (Xu et al., 1990). In a meta-analysis of 9 studies involving 612 subjects and found that the presence of the E4 allele was associated with a significantly greater LDL response to dietary intervention (Masson et al, 2003).

Four studies found significantly different HDL-cholesterol responses between genotype groups: one study found that carriers of the E4 allele had the smallest HDL-cholesterol response, whereas the other 3 studies found that carriers of the $\mathrm{E} 4$ allele had the largest response (Masson et al., 2003).

However, recent evidence strongly suggests that variations in a number of key genes may also be important, including common variants of the Apo E gene. The most convincing evidence to date for genotypic effects on dietary response comes from the extensively studied Apo E gene variant (Lovegrove \& Gitau., 2008).

\subsection{Cholesteryl ester transfer protein (CETP)}

Another gene that affects HDL levels is CETP, encoding for the cholesteryl ester transfer protein that exchanges cholesteryl esters and triglycerids from HDL to other lipoproteins. This protein is also called the "lipid transfer protein:' People with two copies of a common allele at position 279 of this gene tend to have low HDL levels and elevated levels of LDL and VLDL. A variation (279G>A) that decreases plasma levels of CETP is associated with increased HDL levels, decreased LDL and VLDL levels, and a lower risk of cardiovascular disease than the more common (GG) form (DeBusk, 2009; Musunuru., 2010).

A recent meta-analysis which confirms that the I405V and TaqIB variants are indeed associated with lower CETP activity and higher high-density lipoprotein-cholesterol levels (Boekholdt., 2004).

The currently available evidence suggests that several genetic variants in the CETP gene are associated with altered CETP plasma levels and activity, high-density lipoproteincholesterol plasma levels, low-density lipoprotein and high-density lipoprotein particle size, and perhaps the risk of coronary heart disease (Boekholdt., 2004).

\subsection{Hepatic lipase (LIPC)}

Hepatic lipase (HL) is a lipolytic enzyme involved in the hydrolysis of triacylglycerols present in circulating chylomicrons providing nonesterified fatty acids and 2monoacylglycerol for tissue utilization and phospholipids from plasma lipoproteins that participates in metabolizing intermediate-density lipoprotein and large LDL into smaller, denser LDL particles, and in converting HDL2 to HDL3 during reverse cholesterol transport (Ordovas \& Corella., 2004; Fisler, Warden, 2005; Ordovas., 2006; Much et al., 2005). It may suggest a role to play as a ligand for cell-surface proteoglycans in the uptake of lipoproteins by cell-surface receptors (Fisler \& Warden., 2005; Ordovas \& Corella., 2004).

Given the wide spectrum of effects that HL exerts on lipoprotein metabolism, and the significance of the promoter variant (s), it is reasonable to hypothesize that genetic variation at this locus may also be involved in variability in the response to dietary therapy (Ordovas \& Corella., 2004). HL deficiency is characterized by mildly elevated concentrations of triglyceride-rich LDL and HDL particles, as well as impaired metabolism of postprandial triglyceride-rich lipoproteins, which may result in premature atherosclerosis. Conversely, increased HL activity is associated with increased small, dense LDL particles and decreased HDL2 concentrations, which may also result in increased CAD risk. Four common single 
nucleotide polymorphisms (SNPs) on the 5_-flanking region of the HL gene (LIPC) [-763 $(\mathrm{A} / \mathrm{G}),-710(\mathrm{~T} / \mathrm{C}),-514(\mathrm{C} / \mathrm{T})$, and $-250(\mathrm{G} / \mathrm{A})]$ are in total linkage disequilibrium and define a unique haplotype that is associated with variation in HL activity and HDLcholesterol levels (Fisler \& Warden., 2005; Ordovas \& Corella., 2004).

The less common A-allele of the SNP at position-250 is associated with lower HL activity and buoyant LDL particles. Normal and CAD subjects heterozygous for the A-allele have lower HL activity and significantly more buoyant LDL particles. Homozygosity for this allele (AA) is associated with an even lower HL activity. The A-allele is associated with higher HDL2-cholesterol (Ordovas \& Corella., 2004).

An early intervention study with a low-saturated-fat, low-cholesterol diet found that, although significant improvements in fasting lipids occurred, there was no difference in response between genotypes at the hepatic lipase gene (LIPC) polymorphism measured. However, the study of 83 subjects may not have had adequate power to detect a modest effect of genotype (Fisler \& Warden., 2005; Masson \& McNeill., 2005; Ordovas., 2006).

Dietary information collected from Framingham Heart Study participants shows that subjects carrying the CC genotype react to higher contents of fat in their diets by increasing the concentrations of HDL-cholesterol, which could be interpreted as a "defense mechanism" to maintain the homeostasis of lipoprotein metabolism. Conversely, carriers of the TT genotype cannot compensate, and experience decreases on the HDL-cholesterol levels. These data could identify a segment of the population especially susceptible to dietinduced atherosclerosis. Considering the higher frequency of the $\mathrm{T}$ allele among certain ethnic groups (i. e., African-Americans), these data could shed some light on the impaired ability of certain ethnic groups to adapt to new nutritional environments, as clearly seen for Native Americans and Asian Indians. In this regard, they replicated the significant gene-diet interaction demonstrated in the Caucasian population of Framingham in another multiethnic cohort that consisted of Chinese, Malays, and Indians representing the population of Singapore. In addition to the significant gene-diet interactions reported in these papers, these data provides clues about the reasons why genotype-phenotype association studies fail to show consistent results. In theory, this polymorphism at the hepatic lipase gene will show dramatically different outcomes in association studies depending on the dietary environment of the population studies. The impact of these interactions will be magnified in populations with a high prevalence of the T-allele, as it is with Asians and African-Americans (Ordovas \& Corella., 2004).

Three larger observational studies on the effect of a common polymorphism in the LIPC promoter gene $-514 \mathrm{C} \rightarrow \mathrm{T}$ on the response of HDL cholesterol to dietary fat intake have been published. In examining the effects of the $-514 \mathrm{C} \rightarrow \mathrm{T}$ LIPC polymorphism $\mathrm{x}$ dietary fat interaction on HDL in 2130 men and women participating in the Framingham Study, Ordovas et al found that the rarer TT genotype was associated with significantly higher HDL-cholesterol concentrations only in subjects consuming $<30 \%$ of energy from fat. This same interaction was found for saturated and monounsaturated fats but not for polyunsaturated fat. A second association study, in an Asian population of 2170 subjects, found that Asian Indian subjects with a total fat intake of $<30 \%$ of energy and with TT genotype at the $-514 \mathrm{C} \rightarrow \mathrm{T}$ polymorphism had the highest HDL-cholesterol concentrations. This interaction, however, did not apply to the Chinese or Malay subjects in that study, and the significant interactions found for saturated or monounsaturated fats found by Ordovas et al were not found in the study by Tai et al. However, these 2 studies are consistent with 
other studies showing that the $T T$ genotype at $-514 \mathrm{C} \rightarrow \mathrm{T}$ is associated with higher HDL concentrations, although, in 1 of those studies, this effect was attenuated by visceral obesity (Fisler \& Warden., 2005).

The third association study of the interaction between dietary fat and the $-514 \mathrm{C} \rightarrow \mathrm{T}$ polymorphism, by Zhang et al that, from a study population of 18159 men, Zhang et al selected 780 men with confirmed type 2 diabetes. After adjustment for age, smoking, alcohol intake, exercise, and BMI, higher HDL-cholesterol concentrations were found in men with the CT or TT genotype, which is consistent with previous studies. However, they found significantly higher HDL-cholesterol concentrations in men with the CT/TT genotype who consumed large amounts of dietary fat $(\geq 32 \%$ of energy), saturated fat, and monounsaturated fat, a result that is apparently opposite to the findings of the other 2 association studies. Thus, the interaction effect of dietary fat with the $-514 \mathrm{C} \rightarrow \mathrm{T}$ polymorphism was not replicated (Zhang et al., 2005).

Two researcher discussed causes of nonreplication of genetic association studies in obesity and diabetes research that should apply to studies of dyslipidemias as well. An important cause of nonreplication is a lack of statistical power. For any polygenic model, such as models with complex phenotypes (eg, obesity, dyslipidemia, or type 2 diabetes), the effect size for any marker will be small to moderate. Thus, larger sample sizes are needed to ensure adequate power to observe an effect. In the study of Zhang et al, the problem of small sample size (despite the fact that $>18000$ men were screened to identify $\approx 800$ men with type 2 diabetes) is compounded by the fact that the TT genotype is rare, especially in a white population. Thus, only 30 subjects in that study had homozygous $T T$ genotype at $-514 \mathrm{C} \rightarrow \mathrm{T}$ LIPC, and subjects with either the CT or TT genotype were pooled for analysis. Examination of the data of Ordovas et al and Tai et al found that the slope of predicted values for HDL cholesterol versus total fat intake as a percentage of energy is steeply negative in persons with the TT genotype, whereas it is positive for persons with the CT genotype (Ordovas, et al, 2002; Tai et al, 2003). Assuming that the data of Zhang et al followed the same pattern, combining the smaller number $(n=30)$ of persons with the TT genotype with the larger number $(n=247)$ of persons with the CT genotype would mask the effects of percentage of dietary fat and the TT genotype on HDL-cholesterol concentrations. An additional complexity is that BMI and obesity phenotypes may also interact with dietary fat and LIPC genotype to modulate HDL-cholesterol concentrations (Tai et al., 2003; Zhang., 2005). Thus, the nonreplication in the study by Zhang et al of the findings of Ordovas et al and Tai et al is likely due to the small number of persons with the TT genotype who were available in the study of Zhang et al (Fisler \& Warden., 2005).

\subsection{Lipoprotein lipase (LPL)}

LPL, encoding lipoprotein lipase, which hydrolyzes triglycerides in chylomicrons and VLDL particles, converting the latter to LDL particles, as well facilitating cellular lipoprotein uptake (Musunuru, 2010).

Several polymorphisms have been described that disrupt normal LPL function and contribute to the premature development of $\mathrm{CHD}$, primarily through the increased levels of circulating TGs (Much et al., 2005).

Indeed, several of the common LPL polymorphisms described by Merkel et al have recently been established to influence circulating lipid levels in pregnant women, whom are often characterized with high levels of circulating TGs and increased total cholesterol (Merkel et 
al., 2011). Although TG levels were unaffected, certain LPL SNPs modulated HDL levels and may alter the susceptibility of pregnant women to developing CHD. However, further studies are required to definitively define a relationship between lipid levels, CHD, and pregnant women. Therefore, the importance of LPL in the whole-body regulation of lipid metabolism has been avidly demonstrated and merits further exploration.

One common LPL polymorphism, known as T495G Hind III, has been extensively examined and demonstrates the complexity of disease prediction associated with a single SNP. Indeed, preliminary indications suggest that this polymorphism may play a role in the onset of several important diseases, such as CHD, diabetes and obesity. This SNP has been associated with the higher plasma TG and lower HDL levels characteristic with the early onset of diabetes. Preliminary results have also suggested a positive association between the Hind III polymorphism and a predisposition to developing obesity. Finally, this polymorphism has been associated with variations in lipid levels and heart disease, and that these alterations were attenuated by such environmental factors as physical exercise and low calorie diets, reiterating the important interactions arising between lifestyle, nutrition, and disease. Although these associations are not conclusive, they do suggest that LPL variants play a critically important role in the regulation of whole-body lipid metabolism that may predispose an individual to the onset of several metabolic diseases.

A relationship was established between a low calorie diet and the circulating lipid profile in obese individuals with the Hind III polymorphism. Homozygotes $(\mathrm{H} 2 \mathrm{H} 2)$ were found to have significantly higher levels of plasma VLDL-TG and Apo B than heterozygotes. Caloric restriction reduced lipid levels in both $\mathrm{H} 2 \mathrm{H} 2$ and $\mathrm{H} 1$ individuals to a point where no difference was observed between the groups. Although $\mathrm{H} 2 \mathrm{H} 2$ individuals responded more strongly (larger decreases in plasma lipids) to the low calorie diet, these preliminary results identify an important relationship between LPL polymorphisms, function, and diet (Much et al., 2005).

As such, it is difficult to draw any firm conclusions from any one gene-diet study in the absence of replication by another study that examined the same question using similar methodologies. For example, one study demonstrated that a Mediterranean-style, MUFArich diet compared to a high-carbohydrate diet increased LDL size in individuals with certain Apo E gene variants but decreased LDL size in those with other Apo E variants; this is potentially a clinically important observation, but no confirmatory study has yet emerged, calling this observation into doubt. As pointed out by others, the field would greatly benefit from increased collaboration and coordination of studies among international nutrition researchers (Musunuru., 2010).

\section{Magnitude of the response}

Because of the heterogeneity in the type and duration of the interventions described the magnitude of the lipid response to dietary interventions varied widely: in one study the change in LDL cholesterol in the Apo B EcoRI $R-R$ - genotype group was as large as $59 \%$ of the baseline concentration. In the studies that showed a significant difference in response between genotype groups, the results also varied widely: in some studies, the difference in response between 2 genotype groups was $\approx 20 \%$ of the baseline lipid concentration (Rantala et al., 2000; Clitfon., 1997). However, the magnitude of these differences cannot be estimated with any accuracy, largely because most studies had only a small number of subjects in the rare genotype group (Masson et al, 2003). The proportion of variance in the lipid response 
attributable to a single polymorphism is not likely to be $>10 \%$ (Xu et sal., 1990). Therefore, individual genes contribute only a small part to the variation in the lipid response; however, when several genes are considered, the proportion of variance explained could be larger (Masson et al, 2003).

\section{Evidence for a gene-diet interaction}

Evidence suggests that variation in the genes for apolipoproteins A-I, A-IV, B, and E may contribute to the heterogeneity in the lipid response to dietary intervention. Many studies were unable to show significantly different responses between these genotype groups, and the genotypes showing the greatest response are not necessarily consistent between studies. There was insufficient evidence to assess whether lipid responsiveness is affected by variation in the genes for Apo C-III, lipoprotein lipase, hepatic lipase, the cholesteryl ester transfer protein. Although each of these gene products is essential in lipid metabolism, only a handful of studies have investigated variation in these genes, and most of these studies were unable to show significant gene-diet interactions (Masson et al, 2003).

\section{Publication bias}

Publication bias is a problem with any review because "studies with results that are significant, interesting, from large well-funded studies, or of higher quality are more likely to be submitted, published, or published more rapidly than work without such characteristics" (Sutton, 2000). Therefore, it is possible that other relevant dietary intervention studies with genotype information exist but were not included in this review because they have not been published. It is possible that the literature strategy for this review missed studies because the genotype analyses were not mentioned in their title, abstract, or subject headings (Masson et al, 2003).

In the search for explanations for the heterogeneity in lipid responses, reviewers may tend to highlight studies showing significant effects of genetic variation while ignoring a large proportion of studies that found no such results. Studies showing nonsignificant or conflicting results cannot be ignored, especially because they outnumber the studies showing significant effects, notwithstanding the unpublished studies that could have nonsignificant and uninteresting results. Therefore, one has to ask the question "If genetic variability plays a role in the heterogeneity of lipid and lipoprotein responses to dietary change, why have so many studies been unable to demonstrate this with statistical significance?" (Masson et al, 2003).

\section{Possible reasons for conflicting results}

There are many possible reasons why studies have been unable to show statistically significant gene-diet interactions. First, it is highly probable that lipid responses to dietary change are under polygenic control, with each gene contributing a relatively small effect. However, most studies have attempted to find only single-gene effects (Masson et al, 2003). Most of the studies summarized in this review lacked sufficient statistical power to detect any but a very strong effect because the sample sizes were too small, particularly for genotypes with low frequencies of the rare allele. However, many of the studies were retrospective and were not designed to examine gene-diet interactions, but data were 
reexamined after the availability of new information from genotype analyses. Therefore, it is perhaps not surprising that significant effects were not found in many studies because the numbers of individuals in each genotype group were so small. In many studies there were too few subjects homozygous for the rare allele to allow an analysis that would take into account differences in the response between heterozygote's and homozygote's. For Apo E, where there are 6 possible genotypes, differences in the grouping of these could also lead to differences in results between studies. This illustrates that meta-analyses are important because they can detect effects with greater power and greater precision because of their inflated sample size (Rantala et al., 2000; Lopez-Miranda et al., 1994). In addition, in studies with small sample sizes, genotype misclassification of one individual may significantly affect the interpretation and validity of the results. Conflicting results may also occur because of the different dietary protocols that were followed. The studies reviewed varied widely in the composition and length of the baseline and experimental diets. The dietary factors responsible for the changes seen in each genotype group are not clear because many studies modified several dietary factors, and so the dietary content in future studies should be tightly controlled and compliance must be strictly measured not only for cholesterol and the amount and type of fatty acids but also for other influential dietary components such as fiber and plant sterols. In addition, these studies investigated fasting lipid and lipoprotein concentrations; however, the effect of genetic variation may be more evident in the postprandial state than in the less-common fasting state). Differences in the age, sex, body mass index, menopausal status, dietary backgrounds, and baseline lipid values of the participants could also have contributed to the discrepancies between the results. For instance, subjects with the E4 allele tend to have higher baseline total and LDL-cholesterol concentrations, and so greater responses in these subjects could reflect the regression to the mean phenomenon. It is also possible that weight change could account for differences in lipid and lipoprotein changes. In addition, a significant effect may not reflect a causal relation but the allele may be in linkage disequilibrium with another one that does. For example, the base change that results in the Xba I site in the gene for Apo B does not alter the amino acid, and so it may be in linkage disequilibrium with another functional mutation (Masson et al., 2003).

The studies varied widely in terms of the number and type of study participants, the composition and duration of the dietary interventions, the nutrients studied and dietary assessment methods used in the observational studies, and the polymorphisms analyzed some of which had not been studied before with regard to the lipid response to diet (Masson et al., 2005).

\section{Conclusion}

Evidence suggests that genetic variation may contribute to the heterogeneity in lipid responsiveness. At present, the evidence is limited but suggestive and justifies the need for future studies with much larger sample sizes based on power calculations, with carefully controlled dietary interventions, and that investigate the effects of polymorphisms in multiple genes rather than in single genes. Investigating gene-diet interactions will increase our knowledge of the mechanisms involved in lipid metabolism and improve our understanding of the role of diet in reducing cardiovascular disease risk (Masson et al., 2003). 
Future studies will have to be large in order to assess the effects of multiple polymorphisms, and will have to control for many factors other than diet. At present, it is premature to recommend the use of genotyping in the design of therapeutic diets. However, such studies may be useful in identifying the mechanisms by which dietary components influence lipid levels (Masson \& McNeill., 2005).

However, current knowledge is still very limited and so is the potential benefit of its application to clinical practice. Thinking needs to evolve from simple scenarios (e. g., one single dietary component, a single nucleotide polymorphism and risk factor) to more realistic situations involving multiple interactions. One of the first situations where personalized nutrition is likely to be beneficial is in patients with dyslipidemia who require special intervention with dietary treatment. This process could be more efficient if the recommendations were carried out based on genetic and molecular knowledge. Moreover, adherence to dietary advice may increase when it is supported with information based on nutritional genomics, and a patient believes the advice is personalized. However, a number of important changes in the provision of health care are needed to achieve the potential benefits associated with this concept, including a teamwork

approach with greater integration among physicians, food and nutrition professionals, and genetic counselors (Ordovas., 2006).

The ultimate goal of nutritional genomics is to provide sufficient knowledge to allow diagnosis and nutritional treatment recommendations based on an individual's genotype. Defining the interaction effects of nutrients and genes on complex phenotypes will be the challenge of this field of nutrition research for some time to come (Fisler \& Warden, 2005; Gregori et al, 2011; Ordovas \& Corella., 2004).

\section{Ethic}

Although an increased understanding of how these and other genes influence response to nutrients should facilitate the progression of personalized nutrition, the ethical issues surrounding its routine use need careful consideration (Lovegrove \& Gitau., 2008).

The study of nutrigenetics is in its infancy. Many studies published in this area have only considered one SNP in a single gene, with little consideration being given to multiple nutrient-gene-environmental interactions. Although this is scientifically valid, and invaluable for the elucidation of causative mechanisms in disease, multiple gene-nutrientenvironment-gender interactions will be required for developing specific personalized nutritional advice. The collation of data in haplotype databases and biobanks is expensive and difficult to establish, but is a necessity if nutrigenetic research is to progress (Lovegrove \& Gitau., 2008).

Standardized protocols in nutrigenetics are not yet established, the comparison of studies is challenging and conclusions are often difficult to draw. As discussed previously, studies are often retrospective in design and thus of insufficient power to detect nutrient-gene associations. Prospective genotyping increases the power to resolve these associations and should be used whenever possible. With any research, publication bias results in positive associations being reported more often than negative associations. This has applied to nutrigentic studies and created a false impression of the level of significance of many nutrient-gene interactions (Lovegrove \& Gitau., 2008).

There are numerous ethical issues and unavoidable assumptions that need to be considered before personalized nutritional can become routine practice. First, it is important to consider 
whether the genetic tests and personalized food products would be affordable, cost-effective and socially acceptable. It is also of concern that only the well educated and affluent would benefit. The open accessibility of genetic information to third parties has major implications for the availability of health insurance and increased premiums (Lovegrove, Gitau, 2008).

Moreover, it is still unknown whether people will want to undertake genetic tests or even understand the concept of such technology. A survey was conducted by Cogent Research in 2003 on 1000 Americans in which 62\% of respondents reported they had never heard of 'nutrigenomics'. However, if specific products did arise from nutrigenomic research, those interviewed did express interest in an in-depth well-being assessment and also a strong interest in vitamins, fortified foods and natural foods. More research is required to determine whether individuals would want to undergo such tests, and for understanding the value to the individual of an increased awareness of personalized nutrition regimens. There is already a large gap between the existing dietary guidelines and what people actually eat. Knowledge of being at higher than average risk of CVD may motivate people to actually make positive changes to their diets. However, genetic testing could undermine current healthy eating messages, by implying that only those with the 'risky gene' need to eat a healthy diet. These are important unanswered questions that must be addressed if personalized nutritional advice is ever to become part of mainstream disease prevention and treatment. It may be that the interactions between genotype-phenotype and the environment are just too complex to be properly understood from human dietary intervention studies (Lovegrove, Gitau, 2008).

There is resistance to the use and perceived effectiveness of personalized nutrition that is based on genomics, and whether this can offer a solution to diseases caused by a diet that is inappropriate for health (Canon \& Leitzmann, 2005). It has been suggested that it may be more beneficial to use current risk factors as a basis for population screening and the management of CVD (McCluskey et al., 2007). There has also been dialogue on the social, economic and environmental causes of CVD, shifting the emphasis away from dietary intake to food manufacturing as being more effective in disease management (Canon \& Leitzmann, 2005; Lovegrove \& Gitau., 2008).

Progression of knowledge in the fields of nutrient-gene interactions promises a future revolution in preventative health care. However, although there is increasing evidence for interactions between diets/nutrients, genes and environmental factors, there are inconsistencies in the evidence that will limit the application of nutrigenetics in diet-related disease in the immediate future. In addition to the need for adequately powered intervention studies, greater attention should be given to ethical issues, such as the public's acceptance of genetic testing and the economics of this relatively new science (Lovegrove \& Gitau., 2008).

Ethical issues fall into a number of categories: (1) why nutrigenomics? Will it have important public health benefits? (2) questions about research, e. g. concerning the acquisition of information about individual genetic variation; (3) questions about who has access to this information, and its possible misuse; (4) the applications of this information in terms of public health policy, and the negotiation of the potential tension between the interests of the individual in relation to, for example, prevention of conditions such as obesity and allergy; (5) the appropriate ethical approach to the issues, e. g. the moral difference, if any, between therapy and enhancement in relation to individualised diets; whether the 'technological fix' is always appropriate, especially in the wider context of the 
purported lack of public confidence in science, which has special resonance in the sphere of nutrition (Chadwick., 2004).

\section{References}

Amouyel, P. (2000). Genomics and cardiovascular diseases. Bull Acad Natl Med. 184 (7):1431-8.

Boekholdt, SM. \& Kuivenhoven, JA. \& Hovingh, GK. \& Jukema, JW. \& Kastelein, JJ. \& van, Tol A. (2004). CETP gene variation: relation to lipid parameters and cardiovascular risk. Curr Opin Lipidol. 15 (4):393-8.

Campos, H. \& D'Agostino, M. \& Ordovas, JM. (2001). Gene \pm diet interactions and plasma lipoproteins: role of apolipoprotein $\mathrm{E}$ and habitual saturated fat intake. Genet Epidemiol; 20:117 \pm 128 .

Canon, G. \& Leitzmann, C. (2005). The new nutrition science Progect. Public Health Nutr. : 8 (6A):673-694.

Carmena-Ramon, R. \& Ascaso, J F. \& Real, J T. \& Ordovas, J M. (1998 ). Genetic Variation at the ApoA-IV Gene Locus and Response to Diet in Familial Hypercholesterolemia. Arteriosclerosis, Thrombosis, and Vascular Biology. 18:1266- 1274.

Chadwick, R. (2004). Nutrigenomics, individualism and public health. Proc Nutr Soc. 63 (1):161-6.

Clifton, P. \& Kind, K. \& Jones, C. \& , Noakes, M. (1997). Response to dietary fat and cholesterol and genetic polymorphisms. Clin Exp Pharmacol Physiol;24:A21-5.

Corella, D. \& Tucker, K. \& Lahoz, C. \& Coltell, O. \& Cupples, L A. \& Wilson, P WF. \& Schaefer, E J. \& Ordovas, J M. (2001). Alcohol drinking determines the effect of the $A P O E$ locus on LDL-cholesterol concentrations in men: the Framingham Offspring Study. American Journal of Clinical Nutrition. 73 (4): 736-745.

Couch, SC. \& Isasi, CR. \& Karmally. W, et al. (2000). Predictors of postprandial triacylglycerol response in children: the Columbia University Biomarkers Study. Am J Clin Nutr. 72:1119 \pm 1127.

Debra A. K. (2008). Medical Nutrition Therapy For Cardiovascular Disease. In Mahan KL, Escoott- Stump S (eds). Krause's Food Nutrition and Diet Therapy. Suanders. 12th ed. Philadelphia. 833-864. DeBusk, R. . (2008). Nutritional genomic. In Mahan KL, Escoott-Stump S (eds). Krause's Food Nutrition and Diet Therapy. 12th ed. Suanders. Philadelphia. Pp: 364- 382.

DeBusk, R M. (2009). Nutritional Genomics: The Foundation for Personalized Nutrition, What Is Nutritional Genomicsl. In Advanced nutrition and human metabolism. Sareen S. G, Jack L. Smith, James L. G. FIFTH EDITION. WADSWORTH-CENGAGE Learning". USA. pp 29-32.

Dreon, DM. \& Fernstrom, HA. \& Miller, B. \& , Krauss, RM. (1995). Apolipoprotein E isoform phenotype and LDL subclass response to a reduced-fat diet. Arterioscler Thromb Vasc Biol; 15:105 \pm 111 .

El-Sohemy, A. (2007). Nutrigenetics. Forum Nutr. 60:25-30.

Engler, M B. (2009). Nutrigenomics in Cardiovascular Disease: Implications for the Future. Progress in Cardiovascular Nursing. 24 (4): 190-195.

Farhud, D. \& Shalileh, M. (2008). Phenylketonuria and its Dietary Therapy in Children. Iran J Pediatr. 18 (Suppl 1):88-98.

Farhud, DD. \& Zarif Yeganeh, M. \& Zarif Yeganeh, M. (2010). Nutrigenomics and Nutrigenetics. Iranian J Public Health. 39 (4) :1-14. 
Fenech, M. (2008). Genome health nutrigenomics and nutrigenetics-dignosis and nutritional treatment of genome damage on an individual basis. FdChem Tox. 46 (4):1365-70.

Fisler, J S. \& Warden, C H. (2005). Dietary fat and genotype: toward individualized prescriptions for lifestyle changes. American Journal of Clinical Nutrition. 81 (6): 12551256.

Friedlander, Y. \& Leitersdorf, E. \& Vecsler, R. et al. (2000). The contribution of candidate genes to the response of plasma lipids and lipoproteins to dietary challenge. Atherosclerosis. 152:239 \pm 248 .

Frikke-Schmidt R. (2000). Context-dependent and invariant associations between APOE genotype and levels of lipoproteins and risk of ischemic heart disease: a review. Scand J Clin Lab Invest. 60 (Suppl. 233):3 26 .

Gillies, P J. (2003). Nutrigenomics : the Rubicon of molecular nutrition. J am Diet Ass. 103 (12 suppl 2):50-55.

Gregori, D. \& Foltran, F. \& Verduci, E. \& Ballali, S. \& Franchin, L. \& Ghidina, M. \&. Halpern, G M. \& Giovannini, M. (2011) A Genetic Perspective on Nutritional Profiles: Do We Still Need Them?. J Nutrigenet Nutrigenomic. 4 (1):25-35.

Hank Juo, S-H. \& Wyszynski, D F. \& Beaty, T H. \& Huang, H-Y. \& Bailey-W, J E. (1999). Mild association between the A/G polymorphism in the promoter of the apolipoprotein A-I gene and apolipoprotein A-I levels: A meta- analysis. American Journal of Medical Genetics. 82 (3):235-241.

Heilbronn a, L. K., \& Noakes b, M. \& Morris a, A. M. \& Kind b, K. L. \& Clifton, P. M. (2000). 360 His polymorphism of the apolipoproteinA-IV gene and plasma lipid response to energy restricted diets in overweight subjects. Atherosclerosis. 150: 187-192.

Hernell, O. \& West. C. (2008). Do we need personalized recommendations for infants at risk of developing disease? Nestle Nutr Workshop ser Pediatr Program. 62:239-49; discussion 249-52.

Hockey, K. J. \& Anderson, R. A. \& Cook, V. R. \& Hantgan, R. R. \& Weinberg R. B. (2001)Effect of the apolipoprotein A-IV Q360H polymorphism on postprandial plasma triglyceride clearance. J. Lipid Res. . 42: 211-217.

Isasi, CR. \& Couch, SC. \& Deckelbaum, RJ. et al. (2000). The apolipoprotein e2 allele is associated with an anti- atherogenic lipoprotein profile in children: the Columbia University Biomarkers Study. Pediatrics; 106:568 \pm 575 .

Jarvik, GP., \& Goode, EL. \& Austin, MA, et al. (1997). Evidence that the apolipoprotein Egenotype effects on lipid levels can change with age in males: a longitudinal analysis. Am J Hum Genet; 61:171 \pm 181.

Kobayashi, J. \& Saito, Y. \& Taira, K. \& Hikita, M. \& , Takahashi, K. \& Bujo, H. \& Morisaki, N. \& Saito, Y. (2001). Effect of apolipoprotein E3:4 phenotype on postprandial triglycerides and retinyl palmitate metabolism in plasma from hyperlipidemic subjects in Japan. Atherosclerosis. 154; 539-546.

Kusmann, M. \& Fsy, L B. (2008). nutrigenomics and personalized nutrition:science and concept. Personalized Medicine. 5 (5):447-455.

Loktionov, A. \& Scollen, S. \& McKeown, N. \& Bingham S. (2000). Gene \pm nutrient interactions: dietary behavior associated with high coronary heart disease risk particularly affects serum LDL cholesterol in apolipoprotein E (4-carrying freeliving individuals. Br J Nutr. 84:885 \pm 890 . 
Lopez-Miranda, J. \& Ordovas, JM. \& Mata, P, et al. (1994). Effect of apolipoprotein E phenotype on diet-induced lowering of plasma low density lipoprotein cholesterol. J Lipid Res. 35:1965-75.

Lovegrove, J. A. \& Gitau, R. (2008). Personalized nutrition for the prevention of cardiovascular disease: a future perspective. Journal of Human Nutrition and Dietetics. 21 (4) : 306-316.

Mahley, RW. \& PeÂ, pin J. \& Erhan, Paraoglu K, et al. (2000). Low levels of high density lipoproteins in Turks, a population with elevated hepatic lipase: high density lipoprotein characterization and gender-specific effects of apolipoprotein E genotype. J Lipid Res. 41:1290 \pm 1301 .

Masson, L F. \& McNeill, G. \& Avenell, A. (2003). Genetic variation and the lipid response to dietary intervention: a systematic review. American Journal of Clinical Nutrition. 77 (5): 1098-1111.

Masson, L F. \& McNeill, G. (2005). The effect of genetic variation on the lipid response to dietary change: recent findings. Current Opinion in Lipidology. 16 (1):61-67.

McCluskey, S. \& Baker, D. \& Perey, D. \& Lewis, P. \& Middleton, E. (2007). Reduction in cardiovascular risk in association with population screening : a 10- year longitudinal study. J Public Heaht (oxf). 29:379-387.

Merkel, M. \& Eckel, R H. \& Goldberg, I J. (2011). Lipoprotein lipase: genetics, lipid uptake and regulation URL:www. jlr. org. on July 26.

Miggiano, GA. \& De Sanctis, R. (2006). Nutritional genomics: toward a personalized diet. Clin Ter. 157 (4):355-61.

Musunuru, K. (2010). Atherogenic Dyslipidemia: Cardiovascular Risk and Dietary Intervention. Lipids. 45:907-914.

Mutch, D M. \& Wahli, W. \& Gary, W. (2005). Nutrigenomics and nutrigenetics: the emerging faces of nutrition. The FASEB Journal. 19:1602-1616.

Ordovas, J. M. (2002). Gene-diet interaction and plasma lipid responses to dietary intervention. Biochemical Society Transactions. 30: (68-73).

a.Ommen, B V. (2004). Nutrigenomics: Exploiting Systems Biology in the Nutrition and Health Arenas. Nutrition. 20:4-8.

b.Ordovas, JM. (2004). The quest for cardiovascular health in the genomic era : nutrigenetics and plasma lipoproteins. Proc Nutr Soc. 63 (1):145-152.

Ordovas JM, Corella D. (2004). Nutritional genomics. Annu Rev Genomics Hum Genet. 5:71118.

Ordovas JM, Mooser V. (2004). Nutrigenomics and nutrigenetics. Curr Opin Lipidol. 15 (2):101-8.

Ordovas, J. M. (2006). Nutrigenetics, plasma lipids and cardiovascular risk. Journal Of The American Dietetic Association. 106 (7):1074-81.

Ordovas. J M. \& Kaput, J. \& Corella, D. (2007). Nutrition in the genomics era: Cardiovascular disease risk and the Mediterranean diet. Molecular Nutrition $\mathcal{E}$ Food Research. 51 (10): 1293-1299.

Ordovas, J M. (2009). Genetic influences on blood lipids and cardiovascular disease risk: tools for primary prevention. American Journal of Clinical Nutrition. 89 (5):1509S1507S. 
Perez-Martinez, P. \& Garcia-Rios, A. \& Delgado-Lista, J. \& Perez-Jimenez, F. \& LopezMiranda, J. (2011). Nutrigenetics of the postprandial lipoprotein metabolism: evidences from human intervention studies. Curr Vasc Pharmacol. 9 (3):287-91.

Rantala, M. \& Rantala, TT. \& Savolainen, MJ. \& Friedlander, Y. \& Kesaniemi, YA. (2000). Apolipoprotein B gene polymorphisms and serum lipids: meta-analysis of the role of genetic variation in responsiveness to diet. Am J Clin Nutr. 71:713-24.

Rideout, T C. (2011). Getting personal: considering variable interindividual responsiveness to dietary lipid-lowering therapies. Current Opinion in Lipidology. 22 (1):37-42 .

Rimbach, G. \& Minihane, A M. (2009). nutrigenetics and personalized nutrition:how far have we progressed and are we likely to get there? Proceedings of the Nutrition Society. 68 (2): 162-172.

Rubin, J. \& Berglund, L. (2002). Apolipoprotein E and diets: a case of gene-nutrient interaction? Current Opinion in Lipidology. 13:25 \pm 32.

Ryan-Harshman, M. \& Vogel, E. \& Jones-Taggart, H. \& Green-Johnson, J. \& Castle, D. \& Austin, Z. \& Anderson, K. (2008). Nutritional genomics and dietetic professional practice. Kristin Anderson Can J Diet Pract Res. 69 (4):177-82.

Shalileh, M. \& , Shidfar, F. \& Eghtesadi, SH. \& Haghani, H. \& Heidari, I. (2009). The Effects of Calcium Supplement on Serum Lipoprotein in Obese Adults Receiving Energy Restricted Diet. Iranian J Publ Health. 38 (4):10-20.

Shalileh, M. \& , Shidfar, F. \& Haghani, H. \& Eghtesadi, SH. \& Heidari, I. (2010). The influence of calcium supplement on body composition, weight loss and insulin resistance in obese adults receiving low calorie diet. JRMS; 15 (4):1-11.

Sheweta, B. \& Deepak, M. \& BS Khatkar. (2011). Nutrigenomics :The emerging face of nutrition. International Journal of Current Research and Review. 3 (6) :108-113.

Subbiah, M T R. (2007). Nutrigenetics and nutraceuticals: the next wave riding on personalized medicine. Transl res. 149 (2):55-61.

Sutton, A J. \& Duval, S J. \& Tweedie, R L. \& Abrams, K R. \& Jones, D R. (2000). Empirical assessment of effect of publication bias on meta-analyse. BMJ. $320: 1574$

Svacina, S. (2007)Nutrigenetics and nutrigenomics. Cas Lek Cesk. 146 (11):837-9.

Tai, E S. \& Corella, D. \& Deurenberg-Yap, M. \& Cutter, J. \& Chew Kai, S. \& Tan, C E. \& Ordovas, J M. (2003). Dietary Fat Interacts with the -514C $>$ T Polymorphism in the Hepatic Lipase Gene Promoter on Plasma Lipid Profiles in a Multiethnic Asian Population: The 1998 Singapore National Health Survey. J Nutr. 133:3399-3408.

Tikkanen, M. \& Huttunen, JK. \& Pajukanta, PE. \& Pietinen, P. (1995). Apolipoprotein E polymorphism and dietary plasma cholesterol response. Can J Cardiol. 11:93G-96G.

URL: en. wikipedia. org/wiki/Dyslipidemia.

URL : http://www. who. int/mediacentre/factsheets/fs317/en/index. html

Wallace, A J. \& E Humphries, S. \& Fisher, R M. \& Mann, J I. \& Chisholm, A. \& Sutherland, W H F. (2000). Genetic factors associated with response of LDL subfractions to change in the nature of dietary fat. Atherosclerosis. ; 149 (2) : 387-394.

Weggemans, RM. \& Zock, PL. \& Ordovas JM, et al. (2001). Apoprotein E genotype and the response of serum cholesterol to dietary fat, cholesterol and cafestol. Atherosclerosis.

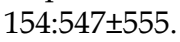

Weinberg, R B. \& Geissinger, B W. \& Kasala, K. \& Hockey, K J. \& James G, T. \& Easter, L. \& Crouse, R. (2000). Effect of apolipoprotein A-IV genotype and dietary fat on cholesterol absorption in humans. J Lipid Res. 41: 2035-2041. 
Weinberg RB. (2002). Apolipoprotein A-IV polymorphisms and diet-gene interactions. Curr Opin Lipidol. 13 (2):125-34.

Wood, Philip A. (2008). Potential of nutrigenetics in the treatment of metabolic disorders. Expert Review of Endocrinology and Metabolism. 3 (6): 705-713.

Xacur-García F, Castillo-Quan JI, Hernández-Escalante VM, Laviada-Molina H. (2008). Nutritional genomics: an approach to the genome-environment interaction. Rev Med Chil. Nov. 136 (11):1460-7.

Xu, CF. \& Boerwinkle, E. \& Tikkanen, MJ. \& Huttunen, JK. \& Humphries, SE. \& Talmud, PJ. (1990). Genetic variation at the apolipoprotein gene loci contribute to response of plasma lipids to dietary change. Genet Epidemiol; 7:261-75.

Zak, A. \& Slaby, A. (2007). Gene - diet interactions in atherogenic dyslipidemias (part1). Cas Lek Cesk. 146 (12):896-901.

Zhang, C. \& Lopez-Ridaura, R. \& Rimm, E B. \& Rifai, Nr. \& Hunter, D J. \& Hu, F B. (2005). Interactions between the $-514 \mathrm{C} \rightarrow \mathrm{T}$ polymorphism of the hepatic lipase gene and lifestyle factors in relation to HDL concentrations among US diabetic men. American Journal of Clinical Nutrition. 81 (6):1429-1435. 


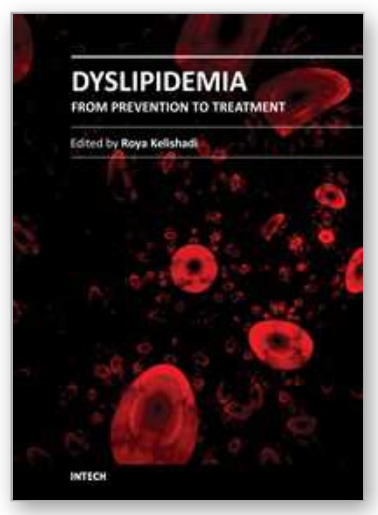

\author{
Dyslipidemia - From Prevention to Treatment \\ Edited by Prof. Roya Kelishadi
}

ISBN 978-953-307-904-2

Hard cover, 468 pages

Publisher InTech

Published online 03, February, 2012

Published in print edition February, 2012

Dyslipidemia has a complex pathophysiology consisting of various genetic, lifestyle, and environmental factors. It has many adverse health impacts, notably in the development of chronic non-communicable diseases.

Significant ethnic differences exist due to the prevalence and types of lipid disorders. While elevated serum total- and LDL-cholesterol are the main concern in Western populations, in other countries hypertriglyceridemia and low HDL-cholesterol are more prevalent. The latter types of lipid disorders are considered as components of the metabolic syndrome. The escalating trend of obesity, as well as changes in lifestyle and environmental factors will make dyslipidemia a global medical and public health threat, not only for adults but for the pediatric age group as well. Several experimental and clinical studies are still being conducted regarding the underlying mechanisms and treatment of dyslipidemia. The current book is providing a general overview of dyslipidemia from diverse aspects of pathophysiology, ethnic differences, prevention, health hazards, and treatment.

\title{
How to reference
}

In order to correctly reference this scholarly work, feel free to copy and paste the following:

Maryam Shalileh (2012). Nutrigenetics and Dyslipidemia, Dyslipidemia - From Prevention to Treatment, Prof. Roya Kelishadi (Ed.), ISBN: 978-953-307-904-2, InTech, Available from:

http://www.intechopen.com/books/dyslipidemia-from-prevention-to-treatment/nutrigenetics-and-dyslipidemia

\section{INTECH}

open science | open minds

\section{InTech Europe}

University Campus STeP Ri

Slavka Krautzeka 83/A

51000 Rijeka, Croatia

Phone: +385 (51) 770447

Fax: +385 (51) 686166

www.intechopen.com

\section{InTech China}

Unit 405, Office Block, Hotel Equatorial Shanghai

No.65, Yan An Road (West), Shanghai, 200040, China

中国上海市延安西路65号上海国际贵都大饭店办公楼 405 单元

Phone: +86-21-62489820

Fax: $+86-21-62489821$ 
(C) 2012 The Author(s). Licensee IntechOpen. This is an open access article distributed under the terms of the Creative Commons Attribution 3.0 License, which permits unrestricted use, distribution, and reproduction in any medium, provided the original work is properly cited. 